\title{
Two Node Tandem Queuing Model with Phase Type Load Dependent Service Having Non-Homogeneous Arrival and Service Processes
}

\author{
Ch.Sreelatha ${ }^{1}$, K. Srinivasa Rao ${ }^{2}$, B.Muniswamy ${ }^{3}$ \\ ${ }^{1,2,3}$ Department of Statistics Andhra University, Visakhapatnam, India
}

\begin{abstract}
This paper addresses the solution procedures for analyzing queuing systems with non-stationary arrival and service processes. Here, it is assumed two queues are connected in tandem and customers arrive to the first node following a non-homogeneous Poisson process. The service processes in both the nodes follow nonhomogeneous Poisson processes. It is further assumed that the customer after completing the service from the first service station may join the second queue or leave the system with certain probability. The service rates in both the service stations are dependent on the number of customers in the queue connected to them. The model behavior is analyzed under transient condition by deriving explicit expressions for the average number of customers in each queue, the waiting time of customers in each queue, the throughput of the nodes, the variability of the number of customers in each queue, the emptiness of the queues. The sensitivity analysis of the model reveals that the nonhomogeneous arrival and service processes can analyze the behavior of the queuing system more accurately under transient conditions. The load dependent service strategy can reduce congestion in queues and avoid delay in transmission. This model also includes some of the earlier queuing models as particular cases.
\end{abstract}

Keywords: Non-stationary queuing model, time dependent queuing model, tandem queuing model, Nonhomogeneous Poisson process, performance measures, sensitivity analysis.

\section{INTRODUCTION:}

Much work has been reported in literature regarding time dependent queuing models. In time dependent models either arrival rate or service rate or both arrival and service rates are dependent on time. This time dependent nature of queuing models has significant applications in communication network modeling (Srinivas rao et.al and suhasini (2014)). The studies made by Abry et al. (2002) and Cappe et al. (2002) on computer network traffic supported that "Modeling and analysis of network traffic using Poisson processes may not serve well in predicting the traffic, since the Poisson like nature assume that the arrival of packets are smoother and less bursty. However, in the present days communication network the aggregate profit does not become smooth and remain bursty". This also supported by the studies made by Leland et al. (1994), Rakesh Singhai et al. (2007) regarding the traffic Ethernet and metropolitan area network (MAN) traffic respectively.

The deviation of arrival and service processes from Poisson process is also studied by Crovella et al. (1997), Murali Krishna et al. (2003), Feldman, A. (2000). The G/M/1 queuing model with weibull inter-arrival distribution is analyzed by Fisher et al. (2001). Newell (1968), Rothkopf and Oren (1979), Massey et al. (1993), Massey and
Whitt (1994), Mandelbaum and Massey (1995), Davis et al. have (1995), William A. Massey (1996), Duffield et al. (2001), William A. Massey (2002), Durga Aparajitha and Raj kumar (2014) and others have studied queuing models with time dependent arrival/service rates. Ward Whitt (2016) has presented an elegant review on time variance single server queues. In all these papers they considered that there is only one single service station. However, in many practical situations one queue is connected to the other queue in tandem.

That is the output of one queue in the input of the other queue. Srinivas rao et al. (2011), Ramasundri et al. (2011), Trinath rao et al. (2012), Srinivas rao et al. (2013), A.V. S Suhasini et al. (2014), Durga aparajitha et al. (2017), Meeravali et al. (2018), Aasish vardan et al. (2019) have developed and analyzed tandem queuing model with time dependent arrival process characterized by nonhomogeneous Poisson process or non-homogeneous compound Poisson process. But they assumed that the service processes in each service station fallows Poisson process. Very little work has been reported in literature work has been reported in literature regarding tandem queuing model with both arrival and service processes are time dependent and fallows a non-homogeneous Poisson process. 


\section{Available online at www.ijrat.org}

Hence, in this paper we develop and analyze two node tandem queuing model with non homogeneous Poisson process for both arrival and service processes. It is also assumed that after getting the service from first service station the customers may join the second queue or leave the system with certain probability. These types of queuing models are known as phase type queuing models. It is also further assumed that the service rate dependent on content of the buffer connected to it. The queue discipline is first-in-first-out and queue capacity is infinity. The behavior of the queuing model is analyzed by deriving the system performance measures explicitly using differencedifferential equations. The sensitivity analysis of the model is performed by changing the input parameters and computing the performance measures. A comparative study of the model is also presented.

\section{QUEUING MODEL}

In this section, we briefly present the development of the queuing model under study. Consider a two node tandem queuing model in which the inter mediatory departures are allowed. The arrival process follows a non-homogeneous Poisson process with mean arrival rate $\lambda(\mathrm{t})=\lambda_{1}+\lambda_{2} \mathrm{t}$. The service processes follow non-homogeneous Poisson processes with mean service rates $\mu_{1}(\mathrm{t})=\alpha_{1}+\beta_{1} \mathrm{t}$ and $\mu_{2}(t)=\alpha_{2}+\beta_{2} t$ for first and second service stations respectively. The service rate is also dependent on the number of customers in the queue. The Queue discipline is first-in-first-out. The buffer capacity is infinity. The probability that the customer leaves the system after first node is $\pi$ and joins the second queue is $\theta=1-\pi$. The schematic diagram representing the queuing model is shown in Figure-1.

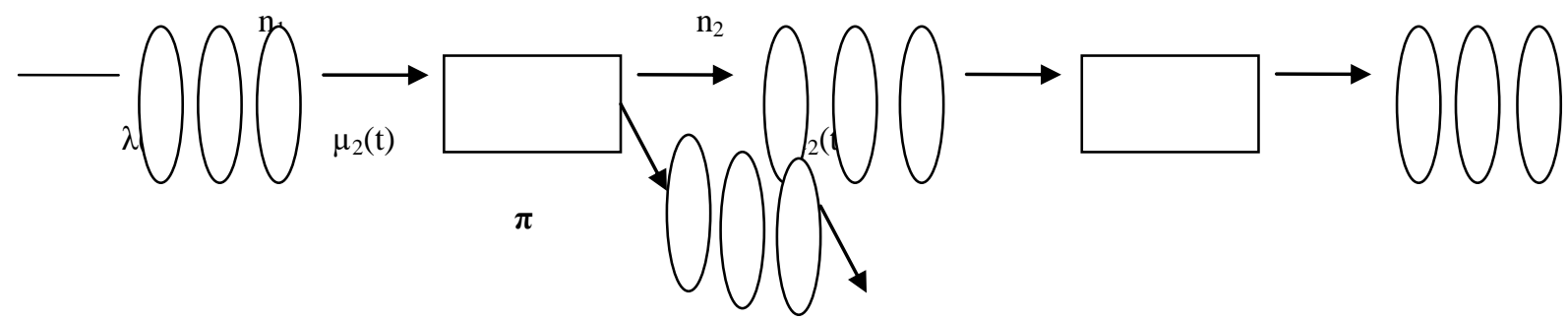

Figure 1: Schematic diagram of the queuing model

Let $\mathrm{n}_{1}$ and $\mathrm{n}_{2}$ denote the number of customers in the first and second queues and $P_{n_{1} n_{2}}(t)$ be the probability that there are $n_{1}$ customers in the first queue and $n_{2}$ customers in the second queue at time ' $t$ '. The difference-differential equations governing the model are:

$$
\begin{aligned}
& \frac{\partial P_{n_{1}, n_{2}}(t)}{\partial t}=-\left(\lambda(t)+n_{1} \mu_{1}(t)+n_{2} \mu_{2}(t)\right) P_{n_{1}, n_{2}}(t)+\lambda(t) P_{n_{1}-1, n_{2}}(t)+\left(n_{1}+1\right) \mu_{1}(t) \theta P_{n_{1}+1, n_{2}-1}(t)+ \\
& \left(n_{1}+1\right) \mu_{1}(t) \pi P_{n_{1}+1, n_{2}}(t)+\left(n_{2}+1\right) \mu_{2}(t) P_{n_{1}, n_{2}+1}(t) ; \quad \forall n_{1}, n_{2} \geq 0 \\
& \frac{\partial P_{n_{1}, 0}(t)}{\partial t}=-\left(\lambda(t)+n_{1} \mu_{1}(t)\right) P_{n_{1,0}}(t)+\lambda(t) P_{n_{1}-1,0}(t)+\left(n_{1}+1\right) \mu_{1}(t) \pi P_{n_{1}+1,0}(t)+\mu_{2}(t) P_{n_{1}, 1}(t) ; \\
& \forall n_{1}>0, n_{2}=0 \\
& \frac{\partial P_{0, n_{2}}(t)}{\partial t}=-\left(\lambda(t)+n_{2} \mu_{2}(t)\right) P_{0, n_{2}}(t)+\mu_{1}(t) \theta P_{1, n_{2}-1}(t)+\mu_{1}(t) \pi P_{1, n_{2}}(t)+\left(n_{2}+1\right) \mu_{2}(t) P_{0, n_{2}+1}(t) ; \\
& \forall n_{2}>0, n_{1}=0 \\
& \frac{\partial P_{0,0}(t)}{\partial t}=-\lambda(t) P_{0,0}(t)+\mu_{1}(t) \pi P_{1,0}(t)+\mu_{2}(t) P_{0,1}(t) ; n_{1}=0, n_{2}=0
\end{aligned}
$$

The probability generating function of $P_{n_{1} n_{2}}(t)$ is

$P\left(S_{1}, S_{2}, t\right)=\sum_{n_{1}=0}^{\infty} \sum_{n_{2}=0}^{\infty} P_{n_{1}, n_{2}}(t) s_{1}^{n_{1}} s_{2}^{n_{2}}$ 


\section{Available online at www.ijrat.org}

Multiplying the equation (1) with $s_{1}^{n_{1}}, s_{2}^{n_{2}}$ and summing over all $\mathrm{n}_{1}, \mathrm{n}_{2}$, we get

$$
\begin{gathered}
\frac{\partial P(t)}{\partial t}=-\sum_{n_{1}=0}^{\infty} \sum_{n_{2}=0}^{\infty}\left(\lambda(t)+n_{1} \mu_{1}(t)+n_{2} \mu_{2}(t)\right) P_{n_{1}, n_{2}}(t) s_{1}^{n_{1}} s_{2}^{n_{2}}+\sum_{n_{1}=0}^{\infty} \sum_{n_{2}=0}^{\infty} \lambda(t) P_{n_{1}-1, n_{2}}(t) s_{1}^{n_{1}} s_{2}^{n_{2}} \\
+\sum_{n_{1}=0}^{\infty} \sum_{n_{2}=0}^{\infty}\left(n_{1}+1\right) \mu_{1}(t) \theta P_{n_{1}+1, n_{2}-1}(t) s_{1}^{n_{1}} s_{2}^{n_{2}}+\sum_{n_{1}=0}^{\infty} \sum_{n_{2}=0}^{\infty}\left(n_{1}+1\right) \mu_{1}(t) \pi P_{n_{1}+1, n_{2}}(t) s_{1}^{n_{1}} s_{2}^{n_{2}} \\
+\sum_{n_{1}=0}^{\infty} \sum_{n_{2}=0}^{\infty}\left(n_{2}+1\right) \mu_{2}(t) P_{n_{1}, n_{2}+1}(t) s_{1}^{n_{1}} s_{2}^{n_{2}}
\end{gathered}
$$

After simplifying, we get

$$
\begin{aligned}
\frac{\partial P\left(S_{1}, S_{2}, t\right)}{\partial t} & =\mu_{1}(t)\left(1-\theta-s_{1}+\theta s_{2}\right) \frac{\partial P\left(s_{1}, s_{2}, t\right)}{\partial s_{1}}+\mu_{2}(t)\left(1-s_{2}\right) \frac{\partial P\left(s_{1}, s_{2}, t\right)}{\partial s_{2}} \\
& -\lambda(t)\left(1-s_{1}\right) P\left(s_{1}, s_{2}, t\right)
\end{aligned}
$$

Solving the equation (4) by Lagrangian's method, the auxiliary equation is

$$
\frac{d t}{1}=\frac{d s_{1}}{-\mu_{1}(t)\left(1-\theta-s_{1}+\theta s_{2}\right)}=\frac{d s_{2}}{-\mu_{2}(t)\left(1-s_{2}\right)}=\frac{d P}{-\lambda(t)\left(1-s_{1}\right) P\left(s_{1}, s_{2}, t\right)}
$$

We have $\lambda(t)=\lambda_{1}+\lambda_{2} t ; \mu_{1}(t)=\alpha_{1}+\beta_{1} t ; 0 \leq \beta_{1} \leq 1$

and $\mu_{2}(t)=\alpha_{2}+\beta_{2} t ; 0 \leq \beta_{2} \leq 1, \alpha_{1} \neq \alpha_{2}$

Solving the first and third terms in equation (5), we get $a=\left(s_{2}-1\right) e^{-\int \mu_{2}(t) d t}$

Solving the first and second terms in equation (5), we get

$$
b=s_{1} e^{-\int \mu_{1}(t) d t}+\left(s_{2}-1\right) e^{-\int \mu_{2}(t) d t} \theta\left(\int \mu_{1}(t) e^{\int\left[\mu_{2}(t)-\mu_{1}(t)\right] d t} d t\right)+\int \mu_{1}(t) e^{-\int \mu_{1}(t) d t} d t
$$

Solving the first and fourth terms in equation (5), we get

$$
\begin{aligned}
c=P\left(s_{1}, s_{2}, t\right) \exp & \left(-\left[s_{1} e^{-\int \mu_{1}(t) d t}+\left(s_{2}-1\right) e^{-\int \mu_{2}(t) d t} \theta\left(\int \mu_{1}(t) e^{\int\left[\mu_{2}(t)-\mu_{1}(t)\right] d t} d t\right)\right.\right. \\
& \left.\left.+\int \mu_{1}(t) e^{-\int \mu_{1}(t) d t} d t\right]\left[\int \lambda(t) e^{\int \mu_{1}(t) d t} d t\right]\right) \\
+ & {\left[\left(s_{2}-1\right) e^{-\int \mu_{2}(t) d t} \theta \int \lambda(t) e^{\int \mu_{1}(t) d t}\left(\int \mu_{1}(t) e^{\int\left[\mu_{2}(t)-\mu_{1}(t)\right] d t} d t\right) d t\right] } \\
& +\left[\int \lambda(t) e^{\int \mu_{1}(t) d t}\left(\int \mu_{1}(t) e^{-\int \mu_{1}(t) d t} d t\right) d t\right]+\int \lambda(t) d t
\end{aligned}
$$

Here $\mathrm{a}, \mathrm{b}$ and $\mathrm{c}$ are arbitrary constants. Using the initial conditions $\mathrm{P}_{00}(0)=1, \mathrm{P}_{00}(\mathrm{t})=0, \forall \mathrm{t}>0$.

The probability generating function of the number of customers in the first queue and the number of customers in the second queue at time ' $\mathrm{t}$ ' is 


\section{Available online at www.ijrat.org}

$$
\begin{aligned}
P\left(s_{1}, s_{2}, t\right)=\exp & \lambda_{1}\left[\left(s_{1}-1\right) e^{-\left(\alpha_{1} t+\beta_{1} \frac{t^{2}}{2}\right)}\left[\frac{\int_{0}^{t}\left(\lambda_{1}+\lambda_{2} \vartheta\right) e^{\left(\alpha_{1} \vartheta+\beta_{1} \frac{\vartheta^{2}}{2}\right)} d \vartheta}{\lambda_{1}}-\frac{1}{\alpha_{1}}\right]\right. \\
& +\theta\left(s_{2}-1\right) e^{-\left(\alpha_{2} t+\beta_{2} \frac{t^{2}}{2}\right)}\left[\frac{1}{\alpha_{2}-\alpha_{1}}-\frac{\int_{0}^{t}\left(\alpha_{1}+\beta_{1} \vartheta\right) e^{\left(\alpha_{2}-\alpha_{1}\right) \vartheta+\left(\beta_{2}-\beta_{1}\right) \frac{\vartheta^{2}}{2}} d \vartheta}{\alpha_{1}}\right] \\
& +\theta\left(s_{2}-1\right) e^{-\left(\alpha_{2} t+\beta_{2} \frac{t^{2}}{2}\right)}\left[\frac{\int_{0}^{t}\left(\lambda_{1}+\lambda_{2} \vartheta\right) e^{\alpha_{1} \vartheta+\beta_{1} \frac{\vartheta^{2}}{2}} d \vartheta \int_{0}^{t}\left(\alpha_{1}+\beta_{1} \vartheta\right) e^{\left(\alpha_{2}-\alpha_{1}\right) \vartheta+\left(\beta_{2}-\beta_{1}\right) \frac{\vartheta^{2}}{2}} d \vartheta}{\lambda_{1}}\right. \\
& \left.\left.-\frac{\int_{0}^{t}\left(\lambda_{1}+\lambda_{2} \vartheta\right) e^{\alpha_{1} \vartheta+\beta_{1} \frac{\vartheta^{2}}{2}}\left(\int_{0}^{t}\left(\alpha_{1}+\beta_{1} \vartheta\right) e^{\left(\alpha_{2}-\alpha_{1}\right) \vartheta+\left(\beta_{2}-\beta_{1} \frac{\vartheta^{2}}{2}\right.} d \vartheta\right) d \vartheta}{\lambda_{1}}-\frac{1}{\alpha_{2}}\right]\right]
\end{aligned}
$$

\section{CHARACTERSTICS OF THE MODEL}

In this section we derive the performance measures of the model expanding $\mathrm{P}\left(\mathrm{s}_{1}, \mathrm{~s}_{2}, \mathrm{t}\right)$ given in equation (9) and collecting the constant terms, we obtain the probability that the queue is empty as

$$
\begin{aligned}
& P_{0,0}(t)=\exp \left[-\lambda_{1}\left[e^{-\left(\alpha_{1} t+\beta_{1} \frac{t^{2}}{2}\right)}\left[\frac{\int_{0}^{t}\left(\lambda_{1}+\lambda_{2} \vartheta\right) e^{\left(\alpha_{1} \vartheta+\beta_{1} \frac{\vartheta^{2}}{2}\right)} d \vartheta}{\lambda_{1}}-\frac{1}{\alpha_{1}}\right]+\theta e^{-\left(\alpha_{2} t+\beta_{2} \frac{t^{2}}{2}\right)}\left[\frac{1}{\alpha_{2}-\alpha_{1}}\right.\right.\right. \\
& \left.-\frac{\int_{0}^{t}\left(\alpha_{1}+\beta_{1} \vartheta\right) e^{\left(\alpha_{2}-\alpha_{1}\right) \vartheta+\left(\beta_{2}-\beta_{1}\right) \frac{\vartheta^{2}}{2}} d \vartheta}{\alpha_{1}}\right] \\
& +\theta e^{-\left(\alpha_{2} t+\beta_{2} \frac{t^{2}}{2}\right)}\left[\frac{\int_{0}^{t}\left(\lambda_{1}+\lambda_{2} \vartheta\right) e^{\alpha_{1} \vartheta+\beta_{1} \frac{\vartheta^{2}}{2}} d \vartheta \int_{0}^{t}\left(\alpha_{1}+\beta_{1} \vartheta\right) e^{\left(\alpha_{2}-\alpha_{1}\right) \vartheta+\left(\beta_{2}-\beta_{1}\right) \frac{\vartheta^{2}}{2}} d \vartheta}{\lambda_{1}}\right. \\
& \left.\left.-\frac{\int_{0}^{t}\left(\lambda_{1}+\lambda_{2} \vartheta\right) e^{\alpha_{1} \vartheta+\beta_{1} \frac{\vartheta^{2}}{2}}\left(\int_{0}^{t}\left(\alpha_{1}+\beta_{1} \vartheta\right) e^{\left(\alpha_{2}-\alpha_{1}\right) \vartheta+\left(\beta_{2}-\beta_{1}\right) \frac{\vartheta^{2}}{2}} d \vartheta\right) d \vartheta}{\lambda_{1}}-\frac{1}{\alpha_{2}}\right]\right]
\end{aligned}
$$

Taking $\mathrm{s}_{2}=1$ in $\mathrm{P}\left(\mathrm{s}_{1}, \mathrm{~s}_{2}, \mathrm{t}\right)$, we obtain the probability generating function of the first queue size as

$\mathrm{P}\left(\mathrm{s}_{1}, \mathrm{t}\right)=\exp \left[\lambda_{1}\left(s_{1}-1\right) e^{-\left(\alpha_{1} t+\beta_{1} \frac{t^{2}}{2}\right)}\left[\frac{\int_{0}^{t}\left(\lambda_{1}+\lambda_{2} \vartheta\right) e^{\left(\alpha_{1} \vartheta+\beta_{1} \frac{\vartheta^{2}}{2}\right)}}{\lambda_{1}}-\frac{1}{\alpha_{1}}\right]\right]$

By expanding $\mathrm{P}\left(\mathrm{s}_{1}, \mathrm{t}\right)$, and collecting the constant terms, we obtain the probability that the first queue is empty as 
$P_{0 .}(t)=\exp \left[-\lambda_{1} e^{-\left(\alpha_{1} t+\beta_{1} \frac{t^{2}}{2}\right)}\left[\frac{\int_{0}^{t}\left(\lambda_{1}+\lambda_{2} \vartheta\right) e^{\left(\alpha_{1} \vartheta+\beta_{1} \frac{\vartheta^{2}}{2}\right)} d \vartheta}{\lambda_{1}}-\frac{1}{\alpha_{1}}\right]\right]$

The mean number of customers in the first queue is

$$
L_{1}(t)=\lambda_{1} e^{-\left(\alpha_{1} t+\beta_{1} \frac{t^{2}}{2}\right)}\left[\frac{\int_{0}^{t}\left(\lambda_{1}+\lambda_{2} \vartheta\right) e^{\left(\alpha_{1} \vartheta+\beta_{1} \frac{\vartheta^{2}}{2}\right)} d \vartheta}{\lambda_{1}}-\frac{1}{\alpha_{1}}\right]
$$

The utilization of the first service station is

$$
\begin{aligned}
U_{1}(t)=1 & -P_{0 .}(t) \\
& =1-\exp \left[-\lambda_{1} e^{-\left(\alpha_{1} t+\beta_{1} \frac{t^{2}}{2}\right)}\left[\frac{\int_{0}^{t}\left(\lambda_{1}+\lambda_{2} \vartheta\right) e^{\left(\alpha_{1} \vartheta+\beta_{1} \frac{\vartheta^{2}}{2}\right)} d \vartheta}{\lambda_{1}}-\frac{1}{\alpha_{1}}\right]\right]
\end{aligned}
$$

The throughput of the first service station is

$$
\begin{aligned}
& T h P_{1}(t)=\mu_{1}(t) U_{1}(t) \\
& =\left(\alpha_{1}+\beta_{1} t\right)\left[1-\exp \left[-\lambda_{1} e^{-\left(\alpha_{1} t+\beta_{1} \frac{t^{2}}{2}\right)}\left[\frac{\int_{0}^{t}\left(\lambda_{1}+\lambda_{2} \vartheta\right) e^{\left(\alpha_{1} \vartheta+\beta_{1} \frac{\vartheta^{2}}{2}\right)} d \vartheta}{\lambda_{1}}-\frac{1}{\alpha_{1}}\right]\right]\right.
\end{aligned}
$$

The average waiting time of a customer in the first system is

$$
\begin{aligned}
& W_{1}(t)=\frac{L_{1}(t)}{T h P_{1}(t)}
\end{aligned}
$$

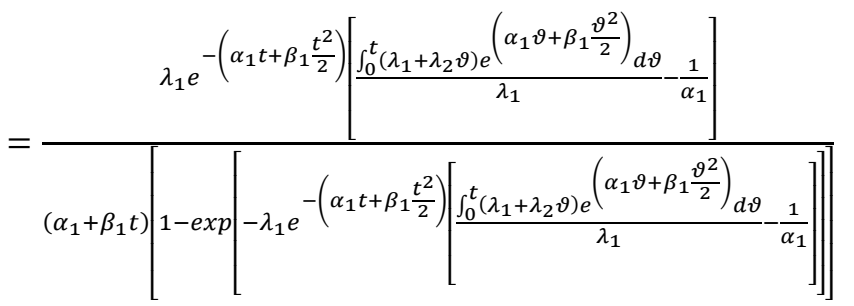

The variance of the number of customers in the first queue is

$V_{1}(t)=\lambda_{1} e^{-\left(\alpha_{1} t+\beta_{1} \frac{t^{2}}{2}\right)}\left[\frac{\int_{0}^{t}\left(\lambda_{1}+\lambda_{2} \vartheta\right) e^{\left(\alpha_{1} \vartheta+\beta_{1} \frac{\vartheta^{2}}{2}\right)} d \vartheta}{\lambda_{1}}-\frac{1}{\alpha_{1}}\right]$

The coefficient of variation of the number of customers in the first queue is

$C V_{1}(t)=\frac{\sqrt{V_{1}(t)}}{L_{1}(t)}=\left[\lambda_{1} e^{-\left(\alpha_{1} t+\beta_{1} \frac{t^{2}}{2}\right)}\left[\frac{\int_{0}^{t}\left(\lambda_{1}+\lambda_{2} \vartheta\right) e^{\left(\alpha_{1} \vartheta+\beta_{1} \frac{\vartheta^{2}}{2}\right)} d \vartheta}{\lambda_{1}}-\frac{1}{\alpha_{1}}\right]\right]^{\frac{-1}{2}}$ 
Taking $\mathrm{s}_{1}=1$ in $\mathrm{P}\left(\mathrm{s}_{1}, \mathrm{~s}_{2}, \mathrm{t}\right)$, we obtain the probability generating function for the Second queue size as

$$
\begin{aligned}
& P\left(s_{2}, t\right)=\exp \left[\lambda _ { 1 } \theta \left[\left(s_{2}-1\right) e^{-\left(\alpha_{2} t+\beta_{2} \frac{t^{2}}{2}\right)}\left[\frac{1}{\alpha_{2}-\alpha_{1}}-\frac{\int_{0}^{t}\left(\alpha_{1}+\beta_{1} \vartheta\right) e^{\left(\alpha_{2}-\alpha_{1}\right) \vartheta+\left(\beta_{2}-\beta_{1}\right) \frac{\vartheta^{2}}{2}} d \vartheta}{\alpha_{1}}\right]+\quad\left(s_{2}-\right.\right.\right. \\
& 1) e^{-\left(\alpha_{2} t+\beta_{2} \frac{t^{2}}{2}\right)}\left[\frac{\int_{0}^{t}\left(\lambda_{1}+\lambda_{2} \vartheta\right) e^{\alpha_{1} \vartheta+\beta_{1} \frac{\vartheta^{2}}{2}} d \vartheta \int_{0}^{t}\left(\alpha_{1}+\beta_{1} \vartheta\right) e^{\left(\alpha_{2}-\alpha_{1}\right) \vartheta+\left(\beta_{2}-\beta_{1}\right) \frac{\vartheta^{2}}{2}} d \vartheta}{\lambda_{1}}-\right. \\
& \left.\left.\left.\frac{\int_{0}^{t}\left(\lambda_{1}+\lambda_{2} \vartheta\right) e^{\alpha_{1} \vartheta+\beta_{1} \frac{\vartheta^{2}}{2}\left(\int_{0}^{t}\left(\alpha_{1}+\beta_{1} \vartheta\right) e^{\left(\alpha_{2}-\alpha_{1}\right) \vartheta+\left(\beta_{2}-\beta_{1}\right) \frac{\vartheta^{2}}{2}} d \vartheta\right) d \vartheta}}{\lambda_{1}}-\frac{1}{\alpha_{2}}\right]\right]\right]
\end{aligned}
$$

By expanding $\mathrm{P}\left(\mathrm{s}_{2}, \mathrm{t}\right)$, and collecting the constant terms, we obtain the probability that the first queue is empty as

$$
\begin{aligned}
& P_{.0}(t)=\exp \left[-\lambda_{1} \theta\left[e^{-\left(\alpha_{2} t+\beta_{2} \frac{t^{2}}{2}\right)}\left[\frac{1}{\alpha_{2}-\alpha_{1}}-\frac{\int_{0}^{t}\left(\alpha_{1}+\beta_{1} \vartheta\right) e^{\left(\alpha_{2}-\alpha_{1}\right) \vartheta+\left(\beta_{2}-\beta_{1}\right) \frac{\vartheta^{2}}{2}} d \vartheta}{\alpha_{1}}\right]\right.\right. \\
&+ e^{-\left(\alpha_{2} t+\beta_{2} \frac{t^{2}}{2}\right)}\left[\frac{\int_{0}^{t}\left(\lambda_{1}+\lambda_{2} \vartheta\right) e^{\alpha_{1} \vartheta+\beta_{1} \frac{\vartheta^{2}}{2}} d \vartheta \int_{0}^{t}\left(\alpha_{1}+\beta_{1} \vartheta\right) e^{\left(\alpha_{2}-\alpha_{1}\right) \vartheta+\left(\beta_{2}-\beta_{1}\right) \frac{\vartheta^{2}}{2}} d \vartheta}{\lambda_{1}}\right. \\
&\left.\left.\left.-\frac{\int_{0}^{t}\left(\lambda_{1}+\lambda_{2} \vartheta\right) e^{\alpha_{1} \vartheta+\beta_{1} \frac{\vartheta^{2}}{2}}\left(\int_{0}^{t}\left(\alpha_{1}+\beta_{1} \vartheta\right) e^{\left(\alpha_{2}-\alpha_{1}\right) \vartheta+\left(\beta_{2}-\beta_{1}\right) \frac{\vartheta^{2}}{2}} d \vartheta\right) d \vartheta}{\lambda_{1}}-\frac{1}{\alpha_{2}}\right]\right]\right]
\end{aligned}
$$

The mean number of customers in the second queue is 
International Journal of Research in Advent Technology, Vol.7, No.3, March 2019 E-ISSN: 2321-9637

\section{Available online at www.ijrat.org}

$$
\begin{aligned}
L_{2}(t)=\lambda_{1} \theta e^{-\left(\alpha_{2} t+\beta_{2} \frac{t^{2}}{2}\right)}\left[\frac{1}{\alpha_{2}-\alpha_{1}}-\frac{\int_{0}^{t}\left(\alpha_{1}+\beta_{1} \vartheta\right) e^{\left(\alpha_{2}-\alpha_{1}\right) \vartheta+\left(\beta_{2}-\beta_{1}\right) \frac{\vartheta^{2}}{2}} d \vartheta}{\alpha_{1}}\right] \\
+\lambda_{1} \theta e^{-\left(\alpha_{2} t+\beta_{2} \frac{t^{2}}{2}\right)}\left[\frac{\int_{0}^{t}\left(\lambda_{1}+\lambda_{2} \vartheta\right) e^{\alpha_{1} \vartheta+\beta_{1} \frac{\vartheta^{2}}{2}} d \vartheta \int_{0}^{t}\left(\alpha_{1}+\beta_{1} \vartheta\right) e^{\left(\alpha_{2}-\alpha_{1}\right) \vartheta+\left(\beta_{2}-\beta_{1}\right) \frac{\vartheta^{2}}{2}} d \vartheta}{\lambda_{1}}\right. \\
\left.+\frac{\int_{0}^{t}\left(\lambda_{1}+\lambda_{2} \vartheta\right) e^{\alpha_{1} \vartheta+\beta_{1} \frac{\vartheta^{2}}{2}}\left(\int_{0}^{t}\left(\alpha_{1}+\beta_{1} \vartheta\right) e^{\left(\alpha_{2}-\alpha_{1}\right) \vartheta+\left(\beta_{2}-\beta_{1}\right) \frac{\vartheta^{2}}{2}} d \vartheta\right) d \vartheta}{\lambda_{1}}-\frac{1}{\alpha_{2}}\right]
\end{aligned}
$$

The utilization of the second service station is

$$
\begin{aligned}
& U_{2}(t)=1-P_{.0}(t) \\
& =1-\exp \left[-\lambda_{1}\left[e^{-\left(\alpha_{2} t+\beta_{2} \frac{t^{2}}{2}\right)}\left[\frac{1}{\alpha_{2}-\alpha_{1}}-\frac{\int_{0}^{t}\left(\alpha_{1}+\beta_{1} \vartheta\right) e^{\left(\alpha_{2}-\alpha_{1}\right) \vartheta+\left(\beta_{2}-\beta_{1}\right) \frac{\vartheta^{2}}{2}} d \vartheta}{\alpha_{1}}\right]\right.\right. \\
& +e^{-\left(\alpha_{2} t+\beta_{2} \frac{t^{2}}{2}\right)}\left[\frac{\int_{0}^{t}\left(\lambda_{1}+\lambda_{2} \vartheta\right) e^{\alpha_{1} \vartheta+\beta_{1} \frac{\vartheta^{2}}{2}} d \vartheta \int_{0}^{t}\left(\alpha_{1}+\beta_{1} \vartheta\right) e^{\left(\alpha_{2}-\alpha_{1}\right) \vartheta+\left(\beta_{2}-\beta_{1}\right) \frac{\vartheta^{2}}{2}} d \vartheta}{\lambda_{1}}\right. \\
& \\
& \left.\left.\left.\quad-\frac{\int_{0}^{t}\left(\lambda_{1}+\lambda_{2} \vartheta\right) e^{\alpha_{1} \vartheta+\beta_{1} \frac{\vartheta^{2}}{2}}\left(\int_{0}^{t}\left(\alpha_{1}+\beta_{1} \vartheta\right) e^{\left(\alpha_{2}-\alpha_{1}\right) \vartheta+\left(\beta_{2}-\beta_{1}\right) \frac{\vartheta^{2}}{2}} d \vartheta\right) d \vartheta}{\lambda_{1}}-\frac{1}{\alpha_{2}}\right]\right]\right]
\end{aligned}
$$

The throughput of the second service station is

$$
\begin{array}{r}
\operatorname{ThP}_{2}(t)=\mu_{2}(t) U_{2}(t) \\
=\left(\alpha_{2}+\beta_{2} t\right)\left[1-\exp \left[-\lambda_{1} \theta\left[e^{-\left(\alpha_{2} t+\beta_{2} \frac{t^{2}}{2}\right)}\left[\frac{1}{\alpha_{2}-\alpha_{1}}-\frac{\int_{0}^{t}\left(\alpha_{1}+\beta_{1} \vartheta\right) e^{\left(\alpha_{2}-\alpha_{1}\right) \vartheta+\left(\beta_{2}-\beta_{1}\right) \frac{\vartheta^{2}}{2}} d \vartheta}{\alpha_{1}}\right]\right.\right.\right. \\
+e^{-\left(\alpha_{2} t+\beta_{2} \frac{t^{2}}{2}\right)}\left[\frac{\int_{0}^{t}\left(\lambda_{1}+\lambda_{2} \vartheta\right) e^{\alpha_{1} \vartheta+\beta_{1} \frac{\vartheta^{2}}{2}} d \vartheta \int_{0}^{t}\left(\alpha_{1}+\beta_{1} \vartheta\right) e^{\left(\alpha_{2}-\alpha_{1}\right) \vartheta+\left(\beta_{2}-\beta_{1}\right) \frac{\vartheta^{2}}{2}} d \vartheta}{\lambda_{1}}\right. \\
\left.\left.\quad-\frac{\int_{0}^{t}\left(\lambda_{1}+\lambda_{2} \vartheta\right) e^{\alpha_{1} \vartheta+\beta_{1} \frac{\vartheta^{2}}{2}}\left(\int_{0}^{t}\left(\alpha_{1}+\beta_{1} \vartheta\right) e^{\left.\left(\alpha_{2}-\alpha_{1}\right) \vartheta+\left(\beta_{2}-\beta_{1}\right) \frac{\vartheta^{2}}{2} d \vartheta\right) d \vartheta}\right.}{\lambda_{1}}-\frac{1}{\alpha_{2}}\right]\right]
\end{array}
$$




\section{Available online at www.ijrat.org}

The average waiting time of a customer in the second system is

$W_{2}(t)=\frac{L_{2}(t)}{T h P_{2}(t)}$, where, $\mathrm{L}_{2}(\mathrm{t})$ and $\mathrm{ThP}_{2}(\mathrm{t})$ are given in equation (21) and (23) respectively

The variance of the number of customers in the Second queue is

$$
\begin{aligned}
& V_{2}(t)=\lambda_{1} \theta e^{-\left(\alpha_{2} t+\beta_{2} \frac{t^{2}}{2}\right)}\left[\frac{1}{\alpha_{2}-\alpha_{1}}-\frac{\int_{0}^{t}\left(\alpha_{1}+\beta_{1} \vartheta\right) e^{\left(\alpha_{2}-\alpha_{1}\right) \vartheta+\left(\beta_{2}-\beta_{1}\right) \frac{\vartheta^{2}}{2}} d \vartheta}{\alpha_{1}}\right] \\
& +\lambda_{1} \theta e^{-\left(\alpha_{2} t+\beta_{2} \frac{t^{2}}{2}\right)}\left[\frac{\int_{0}^{t}\left(\lambda_{1}+\lambda_{2} \vartheta\right) e^{\alpha_{1} \vartheta+\beta_{1} \frac{\vartheta^{2}}{2}} d \vartheta \int_{0}^{t}\left(\alpha_{1}+\beta_{1} \vartheta\right) e^{\left(\alpha_{2}-\alpha_{1}\right) \vartheta+\left(\beta_{2}-\beta_{1}\right) \frac{\vartheta^{2}}{2}} d \vartheta}{\lambda_{1}}\right. \\
& \left.-\frac{\int_{0}^{t}\left(\lambda_{1}+\lambda_{2} \vartheta\right) e^{\alpha_{1} \vartheta+\beta_{1} \frac{\vartheta^{2}}{2}}\left(\int_{0}^{t}\left(\alpha_{1}+\beta_{1} \vartheta\right) e^{\left(\alpha_{2}-\alpha_{1}\right) \vartheta+\left(\beta_{2}-\beta_{1}\right) \frac{\vartheta^{2}}{2}} d \vartheta\right) d \vartheta}{\lambda_{1}}-\frac{1}{\alpha_{2}}\right]
\end{aligned}
$$

The coefficient of variation of the number of customers in the Second queue is

$$
\begin{aligned}
C V_{2}(t)= & {\left[\lambda_{1} \theta e^{-\left(\alpha_{2} t+\beta_{2} \frac{t^{2}}{2}\right)}\left[\frac{1}{\alpha_{2}-\alpha_{1}}-\frac{\int_{0}^{t}\left(\alpha_{1}+\beta_{1} \vartheta\right) e^{\left(\alpha_{2}-\alpha_{1}\right) \vartheta+\left(\beta_{2}-\beta_{1}\right) \frac{\vartheta^{2}}{2}} d \vartheta}{\alpha_{1}}\right]\right.} \\
& +\lambda_{1} \theta e^{-\left(\alpha_{2} t+\beta_{2} \frac{t^{2}}{2}\right)}\left[\frac{\int_{0}^{t}\left(\lambda_{1}+\lambda_{2} \vartheta\right) e^{\alpha_{1} \vartheta+\beta_{1} \frac{\vartheta^{2}}{2}} d \vartheta \int_{0}^{t}\left(\alpha_{1}+\beta_{1} \vartheta\right) e^{\left(\alpha_{2}-\alpha_{1}\right) \vartheta+\left(\beta_{2}-\beta_{1}\right) \frac{\vartheta^{2}}{2} d \vartheta}}{\lambda_{1}}\right. \\
& \left.\left.-\frac{\int_{0}^{t}\left(\lambda_{1}+\lambda_{2} \vartheta\right) e^{\alpha_{1} \vartheta+\beta_{1} \frac{\vartheta^{2}}{2}}\left(\int_{0}^{t}\left(\alpha_{1}+\beta_{1} \vartheta\right) e^{\left.\left(\alpha_{2}-\alpha_{1}\right) \vartheta+\left(\beta_{2}-\beta_{1}\right) \frac{\vartheta^{2}}{2} d \vartheta\right) d \vartheta}\right.}{\lambda_{1}}\right]\right]^{\frac{-1}{2}} \\
& \left.\left.-\frac{1}{\alpha_{2}}\right]\right]^{(26)}
\end{aligned}
$$

The mean number of customers in the queuing system at time $t$ is

$L(t)=L_{1}(t)+L_{2}(t)$

The variance of the number of customers in the system is

$V(t)=V_{1}(t)+V_{2}(t)$

The mean number of customers waiting in system is

$L_{q}(t)=L_{1}(t)-\left(1-P_{0 .}(t)\right)+L_{2}(t)+\left(1-P_{.0}(t)\right)$

\section{NUMERICAL ILLUSTRATION OF THE MODEL}

In this section, the performance of the queuing model is discussed through numerical illustration. Different values of the parameters are considered for service and arrival rates of customers. The transient behavior of the model is studied by computing the performance measures with the following sets of values for the model parameters: $\mathrm{t}=$ 
International Journal of Research in Advent Technology, Vol.7, No.3, March 2019

\author{
E-ISSN: 2321-9637 \\ Available online at www.ijrat.org
}

$0.11,0.13,0.15,0.17 ; \lambda_{1}=4,6,8,9,9.5 ; \lambda_{2}=5,5.5$, $5.9,6.4,6.8 ; \alpha_{1}=9,10,10.2,10.4,10.6 ; \beta_{1}=6,8$, $10,13,16 ; \quad \alpha_{2}=11,11.4,11.8,12.2,12.6 ; \quad \beta_{2}=11$, $15,20,25,30 ; \quad \theta=0.1,0.2,0.3,0.4,0.5 ;$ and $\pi$ $=0.9,0.8,0.7,0.6,0.5$

The probability of emptiness of the queue, the mean number of customers, the utilization of the service station, the throughput of the service station, the variance of the number of customers in the system and the coefficient of variation of the number of customers in the system are computed, for different values of the parameters $t, \lambda_{1}, \lambda_{2}, \alpha_{1}, \beta_{1}, \alpha_{2}$, $\beta_{2}, \theta$ and $\pi$ are presented in Table 1 . The relationship between the parameters and the performance measures are represented in Figures $2 \mathrm{a}$ and $2 \mathrm{~b}$.

From Table 1, it is observed that as time ( $\mathrm{t}$ ) varies from 0.11 to 0.17 , the probability of emptiness of the queuing system decreases from 0.84584 to 0.74183 . Similarly, the probability of emptiness of the first queue decreases from 0.87268 to 0.74922 and the probability of emptiness of the second queue increases from 0.96924 to 0.99013 , the mean number of customers in the first queue increases from 0.13618 to 0.28872 , and in the second queue it decreases from 0.03125 to 0.00992 and the mean number of customers in the system increases from 0.16743 to 0.29864 , when all other parameters are fixed. The probability of emptiness of the system is highly sensitive with respect to time.

It is further observed that as the arrival rate parameter $\left(\lambda_{1}\right)$ varies from 6 to 9 , the probability of emptiness of the queuing system decreases from 0.65331 to 0.53993 . Similarly, the probability of emptiness of the first and second queues decrease from 0.66310 to 0.55212 and 0.98523 to 0.97793 respectively, the mean numbers of customers in the first and second queues increase from 0.41083 to 0.59399 and 0.01488 to 0.02232 respectively, and the mean number of customers in the system increases from 0.42571 to 0.61631 , when all other parameters are fixed.

It is also observed that as the arrival rate parameter $\left(\lambda_{2}\right)$ varies from 5.5 to 6.8 , the probability of emptiness of the queuing system decreases from 0.52073 to 0.51474 . Similarly, the probability of emptiness of the first queue decreases from 0.53314 to 0.52701 , and in the second queue it remains constant, the mean number of customers in the first queue increases from 0.62897 to 0.64054 , and in the second queue it remains constant, the mean number of customers in the system increases from 0.65253 to 0.66410 , when all other parameters are fixed.

It is observed that as the service rate parameter $\left(\alpha_{1}\right)$ varies from 10 to 10.6 , the probability of emptiness of the queuing system decreases from 0.48207 to 0.40084 . Similarly, the probability of emptiness of the first queue increases from 0.52671 to 0.52892 , the probability of emptiness of the second queue decreases from 0.91526 to 0.75785 and the mean number of customers in the first queue decreases from 0.64111 to 0.63692 , the mean number of customers in the second queue increases from 0.08855 to 0.27726 and the mean number of customers in the system increases from 0.72966 to 0.91419 , when all other parameters are fixed.

It is also observed that as the service rate parameter $\left(\beta_{1}\right)$ varies from 8 to 16 , the probability of emptiness of the queuing system increases from 0.40377 to 0.41525 . Similarly, the probability of emptiness of the first queue increases from 0.53272 to 0.54762 , the probability of emptiness of the second queue increases from 0.75795 to 0.75828 and the mean number of customers in the first queue decreases from 0.62977 to 0.60218 , the mean number of customers in the second queue decreases from 0.27714 to 0.27670 and the mean number of customers in the system decreases from 0.90690 to 0.87888 , when all other parameters are fixed.

It is also observed that as the service rate parameter $\left(\beta_{1}\right)$ varies from 8 to 16 , the probability of emptiness of the queuing system increases from 0.40377 to 0.41525 .Similarly, the probability of emptiness of the first queue increases from 0.53272 to 0.54762 , the probability of emptiness of the second queue increases from 0.75795 to 0.75828 and the mean number of customers in the first queue decreases from 0.62977 to 0.60218 , the mean number of customers in the second queue decreases from 0.27714 to 0.27670 and the mean number of customers in the system decreases from 0.90690 to 0.87888 , when all other parameters are fixed. 
International Journal of Research in Advent Technology, Vol.7, No.3, March 2019

E-ISSN: 2321-9637

Available online at www.ijrat.org

Table.1

Values of $P_{00}(t), P_{0 .}(t), P_{.0}(t), L_{1}(t), L_{2}(t)$ for different values of parameters

\begin{tabular}{|c|c|c|c|c|c|c|c|c|c|c|c|c|c|c|}
\hline $\mathbf{t}$ & $\lambda_{1}$ & $\lambda_{2}$ & $\alpha_{1}$ & $\beta_{1}$ & $\boldsymbol{\alpha}_{2}$ & $\overline{\beta_{2}}$ & $\boldsymbol{\theta}$ & $\pi$ & $\mathbf{P}_{00}(t)$ & $\overline{P_{0}(t)}$ & $\mathbf{P}_{.0}(\mathbf{t})$ & $L_{1}(t)$ & $L_{2}(t)$ & $\mathbf{L}(\mathbf{t})$ \\
\hline 0.11 & 4 & 5 & 9 & 6 & 11 & 11 & 0.1 & 0.9 & 0.84584 & 0.87268 & 0.96924 & 0.13618 & 0.03125 & 0.16743 \\
\hline 0.13 & 4 & 5 & 9 & 6 & 11 & 11 & 0.1 & 0.9 & 0.80438 & 0.82222 & 0.97830 & 0.19574 & 0.02194 & 0.21768 \\
\hline 0.15 & 4 & 5 & 9 & 6 & 11 & 11 & 0.1 & 0.9 & 0.77018 & 0.78183 & 0.98510 & 0.24612 & 0.01501 & 0.26113 \\
\hline 0.17 & 4 & 5 & 9 & 6 & 11 & 11 & 0.1 & 0.9 & 0.74183 & 0.74922 & 0.99013 & 0.28872 & 0.00992 & 0.29864 \\
\hline 0.17 & 6 & 5 & 9 & 6 & 11 & 11 & 0.1 & 0.9 & 0.65331 & 0.66310 & 0.98523 & 0.41083 & 0.01488 & 0.42571 \\
\hline 0.17 & 7 & 5 & 9 & 6 & 11 & 11 & 0.1 & 0.9 & 0.61309 & 0.62383 & 0.98279 & 0.47188 & 0.01736 & 0.48924 \\
\hline 0.17 & 8 & 5 & 9 & 6 & 11 & 11 & 0.1 & 0.9 & 0.57535 & 0.58688 & 0.98036 & 0.53294 & 0.01984 & 0.55278 \\
\hline 0.17 & 9 & 5 & 9 & 6 & 11 & 11 & 0.1 & 0.9 & 0.53993 & 0.55212 & 0.97793 & 0.59399 & 0.02232 & 0.61631 \\
\hline 0.17 & 9.5 & 5.5 & 9 & 6 & 11 & 11 & 0.1 & 0.9 & 0.52073 & 0.53314 & 0.97672 & 0.62897 & 0.02356 & 0.65253 \\
\hline 0.17 & 9.5 & 5.9 & 9 & 6 & 11 & 11 & 0.1 & 0.9 & 0.51888 & 0.53125 & 0.97672 & 0.63253 & 0.02356 & 0.65609 \\
\hline 0.17 & 9.5 & 6.4 & 9 & 6 & 11 & 11 & 0.1 & 0.9 & 0.51657 & 0.52889 & 0.97672 & 0.63698 & 0.02356 & 0.66054 \\
\hline 0.17 & 9.5 & 6.8 & 9 & 6 & 11 & 11 & 0.1 & 0.9 & 0.51474 & 0.52701 & 0.97672 & 0.64054 & 0.02356 & 0.66410 \\
\hline 0.17 & 9.5 & 6.8 & 10 & 6 & 11 & 11 & 0.1 & 0.9 & 0.48207 & 0.52671 & 0.91526 & 0.64111 & 0.08855 & 0.72966 \\
\hline 0.17 & 9.5 & 6.8 & 10.2 & 6 & 11 & 11 & 0.1 & 0.9 & 0.46754 & 0.52728 & 0.88671 & 0.64002 & 0.12024 & 0.76026 \\
\hline 0.17 & 9.5 & 6.8 & 10.4 & 6 & 11 & 11 & 0.1 & 0.9 & 0.44426 & 0.52802 & 0.84136 & 0.63862 & 0.17274 & 0.81135 \\
\hline 0.17 & 9.5 & 6.8 & 10.6 & 6 & 11 & 11 & 0.1 & 0.9 & 0.40084 & 0.52892 & 0.75785 & 0.63692 & 0.27726 & 0.91419 \\
\hline 0.17 & 9.5 & 6.8 & 10.6 & 8 & 11 & 11 & 0.1 & 0.9 & 0.40377 & 0.53272 & 0.75795 & 0.62977 & 0.27714 & 0.90690 \\
\hline 0.17 & 9.5 & 6.8 & 10.6 & 10 & 11 & 11 & 0.1 & 0.9 & 0.40688 & 0.53649 & 0.75804 & 0.62271 & 0.27702 & 0.89973 \\
\hline 0.17 & 9.5 & 6.8 & 10.6 & 13 & 11 & 11 & 0.1 & 0.9 & 0.41099 & 0.54209 & 0.75817 & 0.61233 & 0.27685 & 0.88919 \\
\hline 0.17 & 9.5 & 6.8 & 10.6 & 16 & 11 & 11 & 0.1 & 0.9 & 0.41525 & 0.54762 & 0.75828 & 0.60218 & 0.27670 & 0.87888 \\
\hline 0.17 & 9.5 & 6.8 & 10.6 & 16 & 11.4 & 11 & 0.1 & 0.9 & 0.48949 & 0.54762 & 0.89386 & 0.60218 & 0.11221 & 0.71438 \\
\hline 0.17 & 9.5 & 6.8 & 10.6 & 16 & 11.8 & 11 & 0.1 & 0.9 & 0.51628 & 0.54762 & 0.94277 & 0.60218 & 0.05893 & 0.66111 \\
\hline 0.17 & 9.5 & 6.8 & 10.6 & 16 & 12.2 & 11 & 0.1 & 0.9 & 0.52966 & 0.54762 & 0.96721 & 0.60218 & 0.03334 & 0.63552 \\
\hline 0.17 & 9.5 & 6.8 & 10.6 & 16 & 12.6 & 11 & 0.1 & 0.9 & 0.53745 & 0.54762 & 0.98144 & 0.60218 & 0.01874 & 0.62091 \\
\hline 0.17 & 9.5 & 6.8 & 10.6 & 16 & 12.6 & 15 & 0.1 & 0.9 & 0.53826 & 0.54762 & 0.98291 & 0.60218 & 0.01724 & 0.61942 \\
\hline 0.17 & 9.5 & 6.8 & 10.6 & 16 & 12.6 & 20 & 0.1 & 0.9 & 0.53919 & 0.54762 & 0.98462 & 0.60218 & 0.01550 & 0.61768 \\
\hline 0.17 & 9.5 & 6.8 & 10.6 & 16 & 12.6 & 25 & 0.1 & 0.9 & 0.54006 & 0.54762 & 0.98620 & 0.60218 & 0.0139 & 0.61608 \\
\hline 0.17 & 9.5 & 6.8 & 10.6 & 16 & 12.6 & 30 & 0.1 & 0.9 & 0.54086 & 0.54762 & 0.98765 & 0.60218 & 0.01242 & 0.61460 \\
\hline 0.17 & 9.5 & 6.8 & 10.6 & 16 & 12.6 & 30 & 0.2 & 0.8 & 0.53418 & 0.54762 & 0.97546 & 0.60218 & 0.02485 & 0.62702 \\
\hline 0.17 & 9.5 & 6.8 & 10.6 & 16 & 12.6 & 30 & 0.3 & 0.7 & 0.52758 & 0.54762 & 0.96342 & 0.60218 & 0.03727 & 0.63945 \\
\hline 0.17 & 9.5 & 6.8 & 10.6 & 16 & 12.6 & 30 & 0.4 & 0.6 & 0.52107 & 0.54762 & 0.95152 & 0.60218 & 0.04969 & 0.65187 \\
\hline 0.17 & 9.5 & 6.8 & 10.6 & 16 & 12.6 & 30 & 0.5 & 0.5 & 0.51464 & 0.54762 & 0.93977 & 0.60218 & 0.06212 & 0.66429 \\
\hline
\end{tabular}

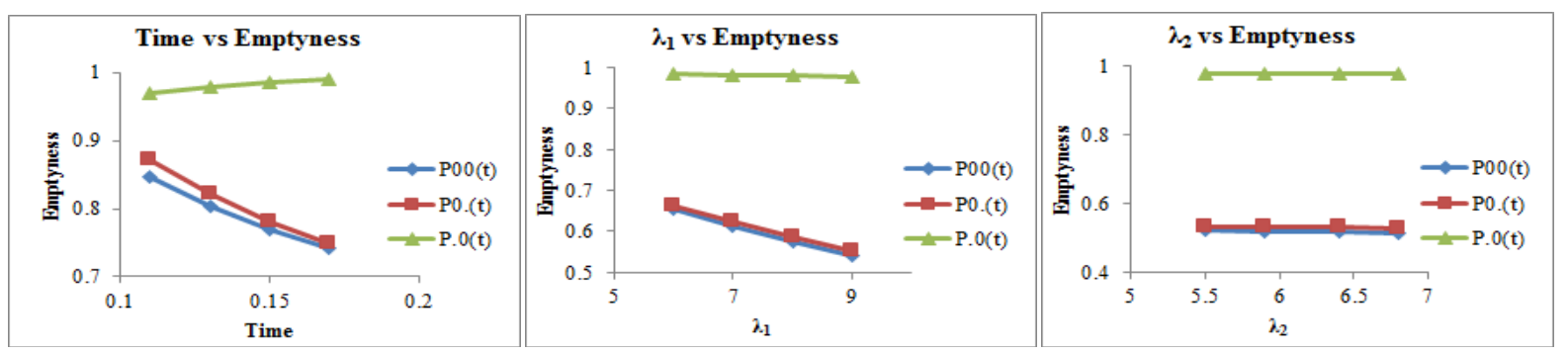




\section{Available online at www.ijrat.org}
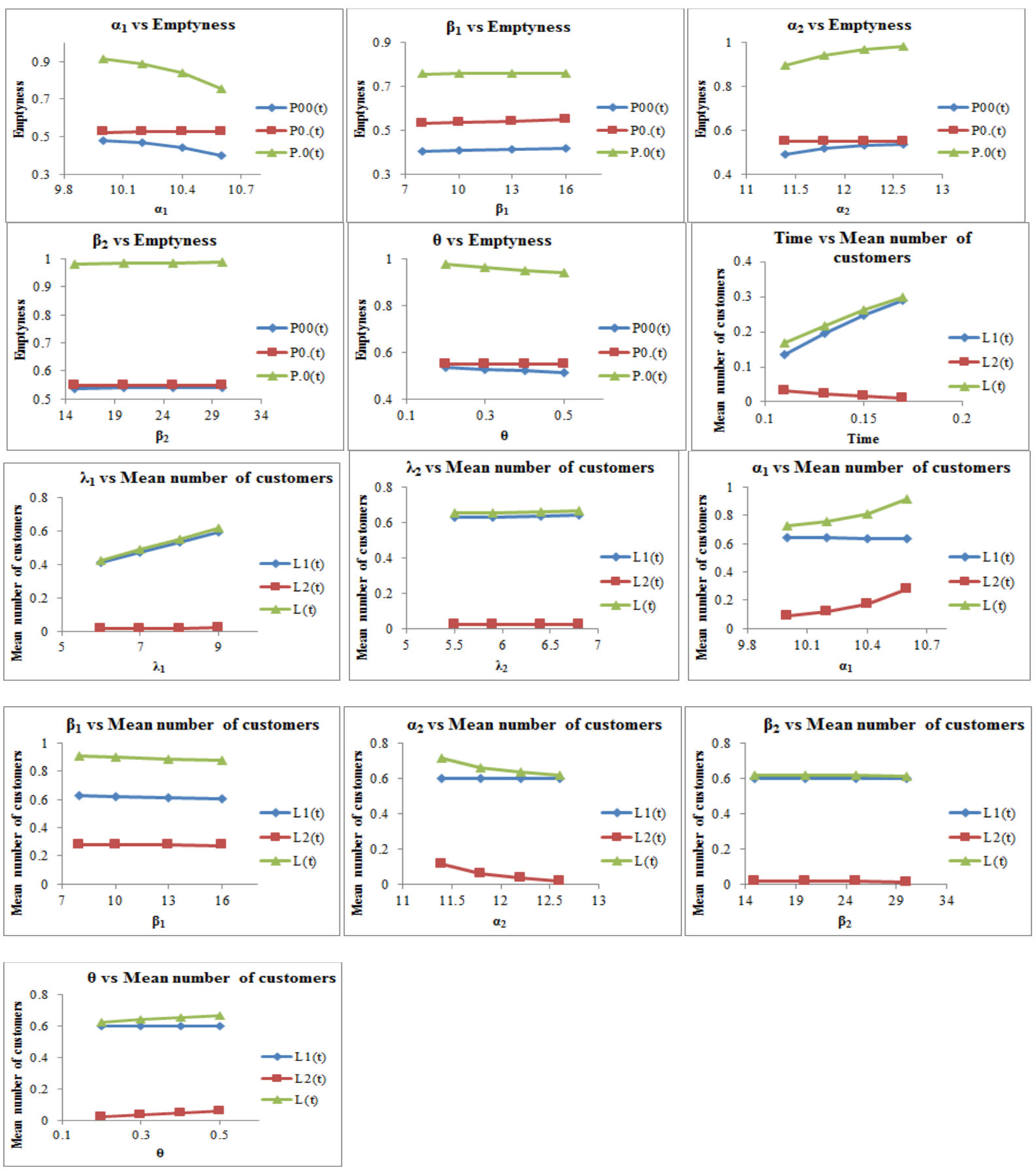

Figure 2a: The relationship between the parameters and performance measures.

It is observed that as the service rate parameter $\left(\alpha_{2}\right)$ varies from 11.4 to 12.6 , the probability of emptiness of the queuing system increases from 0.48949 to 0.53745 . Similarly, the probability of emptiness of the second queue increases from 0.89386 to 0.98144 , and in the first queue it remains constant, the mean number of customers in the second queue decreases from
0.11221 to 0.01874 , and in the first queue it remains constant, the mean number of customers in the system decreases from 0.71438 to 0.62091 , when all other parameters are fixed.

It is also observed that as the service rate parameter $\left(\beta_{2}\right)$ varies from 15 to 30 , the probability of emptiness of the queuing system increases from 0.53826 to 0.54086 . Similarly, the probability of 
International Journal of Research in Advent Technology, Vol.7, No.3, March 2019

E-ISSN: 2321-9637

Available online at www.ijrat.org

emptiness of the second queue increases from 0.98291 to 0.98765 , and in the first queue it remains constant, the mean number of customers in the second queue decreases from 0.01724 to 0.01242 , and in the first queue it remains constant, the mean number of customers in the system decreases from 0.61942 to 0.61460 , when all other parameters are fixed.

It is also observed that as the service rate parameter $(\theta)$ varies from 0.2 to 0.5 , the probability of emptiness of the system decreases from 0.53418 to 0.51464. Similarly, the probability of emptiness of the second queue decreases from 0.97546 to 0.93977 , and in the first queue it remains constant, the mean number of customers in the second queue increases from 0.02485 to 0.06212 , and in the first queue it remains constant, the mean number of customers in the system increases from 0.62702 to 0.66429 , when all other parameters are fixed.

From Table 2, it is observed that as time (t) varies from 0.11 to 0.17 , the utilization of the first service station, the throughput of the first service station, and the average waiting time of a customer in the first queue increase from 0.12732 to 0.25078 , 1.22987 to 2.51280 and 0.11073 to 0.11490 respectively. But in the second queue they decrease from 0.03076 to $0.00987,0.37561$ to 0.12703 and 0.08319 to 0.07809 respectively, when all other parameters are fixed. It is observed that the utilization of the service stations, throughput of the service stations and the waiting time of customers in each queue are highly sensitive with respect to time.
It is further observed that as the arrival rate parameter $\left(\lambda_{1}\right)$ varies from 6 to 9 , the utilization of the service station, the throughput of service station, and the average waiting time of a customers in the first and second queue increase from 0.33690 to $0.44788,0.01477$ to $0.02207,3.37574$ to 4.48778 , 0.19007 to $0.28405,0.12170$ to 0.13236 and 0.07828 to 0.07857 respectively, when all other parameters are fixed.

It is also observed that as the arrival rate parameter $\left(\lambda_{2}\right)$ varies from 5.5 to 6.8 , the utilization of the first service station, the throughput of the first service station, and the average waiting time of customers in the first queue increase from 0.46686 to $0.47299,4.67794$ to 4.73939 and 0.13445 to 0.13515 respectively. And the performance measures remain constant in second queue, when all other parameters are fixed.

It is observed that as the service rate parameter $\left(\alpha_{1}\right)$ varies from 10 to 10.6 , the utilization of the first service station decreases from 0.47329 to 0.47108 , the utilization of the second service station increases from 0.08474 to 0.24215 , the throughput of the first and second service stations increase from 5.21569 to $5.47398,1.09060$ to 3.11642 respectively, and the average waiting time of a customers in the first queue decreases from 0.12292 to 0.11635 , the average waiting time of a customers in the second queue increases from 0.08119 to 0.08897 , when all other parameters are fixed.

Table 2

Values of $U_{1}(t), U_{2}(t), T h p_{1}(t), T h p_{2}(t), W_{1}(t), W_{2}(t)$ for different values of parameters

\begin{tabular}{|c|l|l|l|l|l|l|l|l|l|l|l|l|l|l|}
\hline $\mathbf{t}$ & $\boldsymbol{\lambda}_{\mathbf{1}}$ & $\boldsymbol{\lambda}_{\mathbf{2}}$ & $\boldsymbol{\alpha}_{\mathbf{1}}$ & $\boldsymbol{\beta}_{\mathbf{1}}$ & $\boldsymbol{\alpha}_{\mathbf{1}}$ & $\boldsymbol{\beta}_{\mathbf{2}}$ & $\boldsymbol{\theta}$ & $\boldsymbol{\pi}$ & $\mathbf{U}_{\mathbf{1}}(\mathbf{t})$ & $\mathbf{U}_{\mathbf{2}}(\mathbf{t})$ & $\mathbf{T h p}_{\mathbf{1}}(\mathbf{t})$ & $\mathbf{T h p}_{\mathbf{2}}(\mathbf{t})$ & $\mathbf{W}_{\mathbf{1}}(\mathbf{t})$ & $\mathbf{W}_{\mathbf{2}}(\mathbf{t})$ \\
\hline $\mathbf{0 . 1 1}$ & 4 & 5 & 9 & 6 & 11 & 11 & 0.1 & 0.9 & 0.12732 & 0.03076 & 1.22987 & 0.37561 & 0.11073 & 0.08319 \\
\hline $\mathbf{0 . 1 3}$ & 4 & 5 & 9 & 6 & 11 & 11 & 0.1 & 0.9 & 0.17778 & 0.02170 & 1.73866 & 0.26972 & 0.11258 & 0.08134 \\
\hline $\mathbf{0 . 1 5}$ & 4 & 5 & 9 & 6 & 11 & 11 & 0.1 & 0.9 & 0.21817 & 0.01490 & 2.15987 & 0.18844 & 0.11395 & 0.07965 \\
\hline $\mathbf{0 . 1 7}$ & 4 & 5 & 9 & 6 & 11 & 11 & 0.1 & 0.9 & 0.25078 & 0.00987 & 2.51280 & 0.12703 & 0.11490 & 0.07809 \\
\hline 0.17 & $\mathbf{6}$ & 5 & 9 & 6 & 11 & 11 & 0.1 & 0.9 & 0.33690 & 0.01477 & 3.37574 & 0.19007 & 0.12170 & 0.07828 \\
\hline 0.17 & $\mathbf{7}$ & 5 & 9 & 6 & 11 & 11 & 0.1 & 0.9 & 0.37617 & 0.01721 & 3.76927 & 0.22147 & 0.12519 & 0.07838 \\
\hline 0.17 & $\mathbf{8}$ & 5 & 9 & 6 & 11 & 11 & 0.1 & 0.9 & 0.41312 & 0.01964 & 4.13949 & 0.25280 & 0.12875 & 0.07847 \\
\hline 0.17 & $\mathbf{9}$ & 5 & 9 & 6 & 11 & 11 & 0.1 & 0.9 & 0.44788 & 0.02207 & 4.48778 & 0.28405 & 0.13236 & 0.07857 \\
\hline 0.17 & 9.5 & $\mathbf{5 . 5}$ & 9 & 6 & 11 & 11 & 0.1 & 0.9 & 0.46686 & 0.02328 & 4.67794 & 0.29964 & 0.13445 & 0.07862 \\
\hline 0.17 & 9.5 & $\mathbf{5 . 9}$ & 9 & 6 & 11 & 11 & 0.1 & 0.9 & 0.46875 & 0.02328 & 4.69692 & 0.29964 & 0.13467 & 0.07862 \\
\hline 0.17 & 9.5 & $\mathbf{6 . 4}$ & 9 & 6 & 11 & 11 & 0.1 & 0.9 & 0.47111 & 0.02328 & 4.72056 & 0.29964 & 0.13494 & 0.07862 \\
\hline 0.17 & 9.5 & $\mathbf{6 . 8}$ & 9 & 6 & 11 & 11 & 0.1 & 0.9 & 0.47299 & 0.02328 & 4.73939 & 0.29964 & 0.13515 & 0.07862 \\
\hline 0.17 & 9.5 & 6.8 & $\mathbf{1 0}$ & 6 & 11 & 11 & 0.1 & 0.9 & 0.47329 & 0.08474 & 5.21569 & 1.09060 & 0.12292 & 0.08119 \\
\hline 0.17 & 9.5 & 6.8 & $\mathbf{1 0 . 2}$ & 6 & 11 & 11 & 0.1 & 0.9 & 0.47272 & 0.11329 & 5.30391 & 1.45807 & 0.12067 & 0.08246 \\
\hline 0.17 & 9.5 & 6.8 & $\mathbf{1 0 . 4}$ & 6 & 11 & 11 & 0.1 & 0.9 & 0.47198 & 0.15864 & 5.38999 & 2.04171 & 0.11848 & 0.08460 \\
\hline 0.17 & 9.5 & 6.8 & $\mathbf{1 0 . 6}$ & 6 & 11 & 11 & 0.1 & 0.9 & 0.47108 & 0.24215 & 5.47398 & 3.11642 & 0.11635 & 0.08897 \\
\hline 0.17 & 9.5 & 6.8 & 10.6 & $\mathbf{8}$ & 11 & 11 & 0.1 & 0.9 & 0.46728 & 0.24205 & 5.58871 & 3.11521 & 0.11269 & 0.08896 \\
\hline
\end{tabular}


International Journal of Research in Advent Technology, Vol.7, No.3, March 2019

E-ISSN: 2321-9637

\section{Available online at www.ijrat.org}

\begin{tabular}{|l|l|l|l|l|l|l|l|l|l|l|l|l|l|l|}
\hline 0.17 & 9.5 & 6.8 & 10.6 & $\mathbf{1 0}$ & 11 & 11 & 0.1 & 0.9 & 0.46351 & 0.24196 & 5.70122 & 3.11404 & 0.10922 & 0.08896 \\
\hline 0.17 & 9.5 & 6.8 & 10.6 & $\mathbf{1 3}$ & 11 & 11 & 0.1 & 0.9 & 0.45791 & 0.24183 & 5.86588 & 3.11241 & 0.10439 & 0.08895 \\
\hline 0.17 & 9.5 & 6.8 & 10.6 & $\mathbf{1 6}$ & 11 & 11 & 0.1 & 0.9 & 0.45238 & 0.24172 & 6.02573 & 3.11092 & 0.09993 & 0.08895 \\
\hline 0.17 & 9.5 & 6.8 & 10.6 & 16 & $\mathbf{1 1 . 4}$ & 11 & 0.1 & 0.9 & 0.45238 & 0.10614 & 6.02573 & 1.40847 & 0.09993 & 0.07966 \\
\hline 0.17 & 9.5 & 6.8 & 10.6 & 16 & $\mathbf{1 1 . 8}$ & 11 & 0.1 & 0.9 & 0.45238 & 0.05723 & 6.02573 & 0.78231 & 0.09993 & 0.07533 \\
\hline 0.17 & 9.5 & 6.8 & 10.6 & 16 & $\mathbf{1 2 . 2}$ & 11 & 0.1 & 0.9 & 0.45238 & 0.03279 & 6.02573 & 0.46136 & 0.09993 & 0.07226 \\
\hline 0.17 & 9.5 & 6.8 & 10.6 & 16 & $\mathbf{1 2 . 6}$ & 11 & 0.1 & 0.9 & 0.45238 & 0.01856 & 6.02573 & 0.26859 & 0.09993 & 0.06976 \\
\hline 0.17 & 9.5 & 6.8 & 10.6 & 16 & 12.6 & $\mathbf{1 5}$ & 0.1 & 0.9 & 0.45238 & 0.01709 & 6.02573 & 0.25897 & 0.09993 & 0.06658 \\
\hline 0.17 & 9.5 & 6.8 & 10.6 & 16 & 12.6 & $\mathbf{2 0}$ & 0.1 & 0.9 & 0.45238 & 0.01538 & 6.02573 & 0.24614 & 0.09993 & 0.06299 \\
\hline 0.17 & 9.5 & 6.8 & 10.6 & 16 & 12.6 & $\mathbf{2 5}$ & 0.1 & 0.9 & 0.45238 & 0.01380 & 6.02573 & 0.23261 & 0.09993 & 0.05976 \\
\hline 0.17 & 9.5 & 6.8 & 10.6 & 16 & 12.6 & $\mathbf{3 0}$ & 0.1 & 0.9 & 0.45238 & 0.01235 & 6.02573 & 0.21853 & 0.09993 & 0.05685 \\
\hline 0.17 & 9.5 & 6.8 & 10.6 & 16 & 12.6 & 30 & 0.2 & 0.8 & 0.45238 & 0.02454 & 6.02573 & 0.43437 & 0.09993 & 0.05720 \\
\hline 0.17 & 9.5 & 6.8 & 10.6 & 16 & 12.6 & 30 & 0.3 & 0.7 & 0.45238 & 0.03658 & 6.02573 & 0.64754 & 0.09993 & 0.05756 \\
\hline 0.17 & 9.5 & 6.8 & 10.6 & 16 & 12.6 & 30 & 0.4 & 0.6 & 0.45238 & 0.04848 & 6.02573 & 0.85808 & 0.09993 & 0.05791 \\
\hline 0.17 & 9.5 & 6.8 & 10.6 & 16 & 12.6 & 30 & 0.5 & 0.5 & 0.45238 & 0.06023 & 6.02573 & 1.06602 & 0.09993 & 0.05827 \\
\hline
\end{tabular}
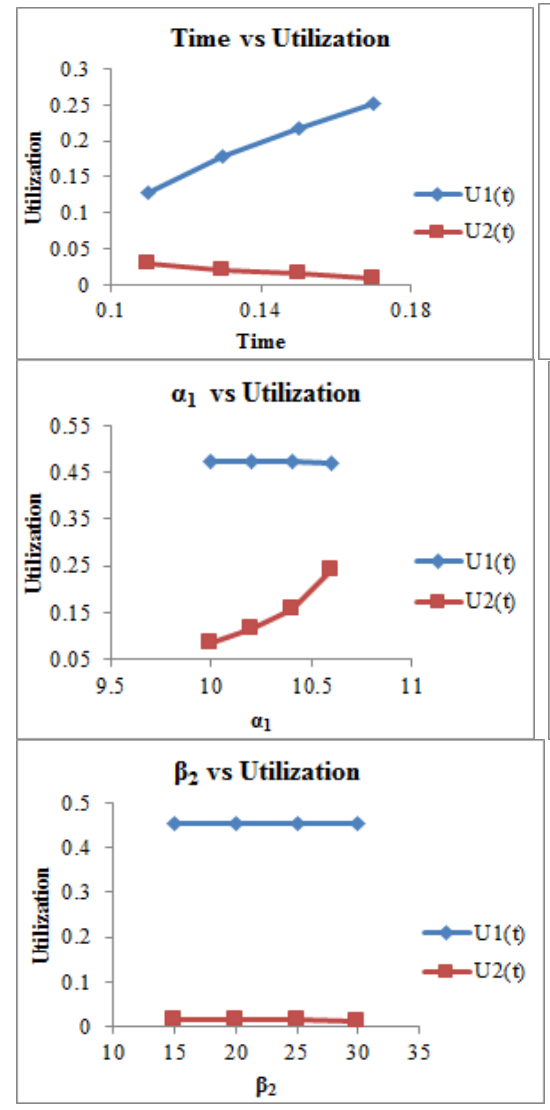
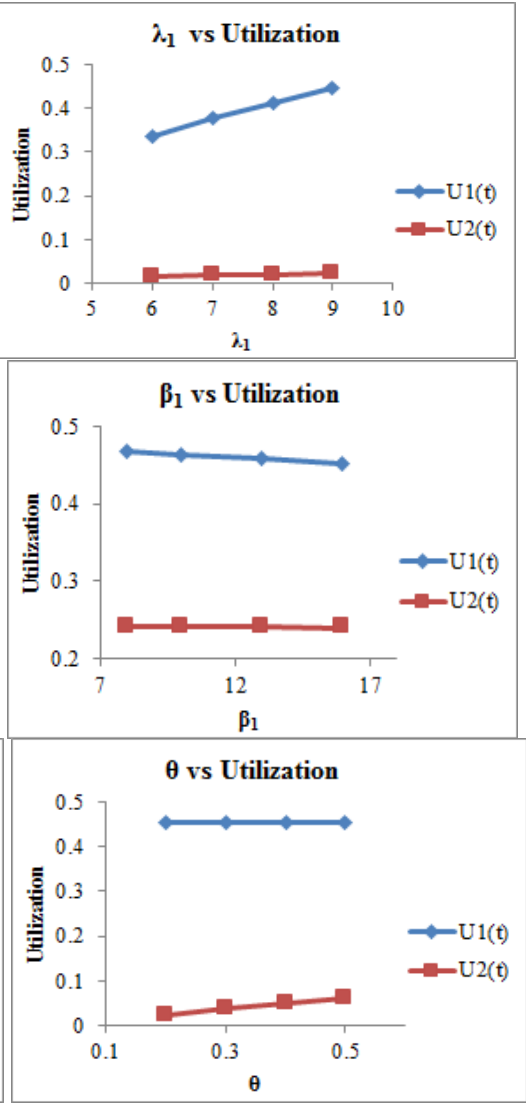
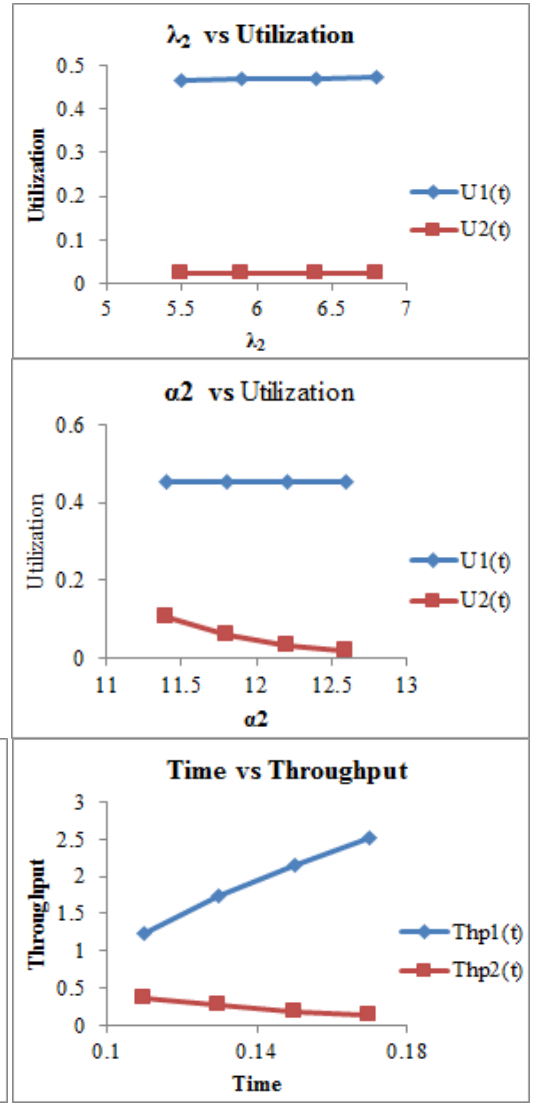


\section{Available online at www.ijrat.org}

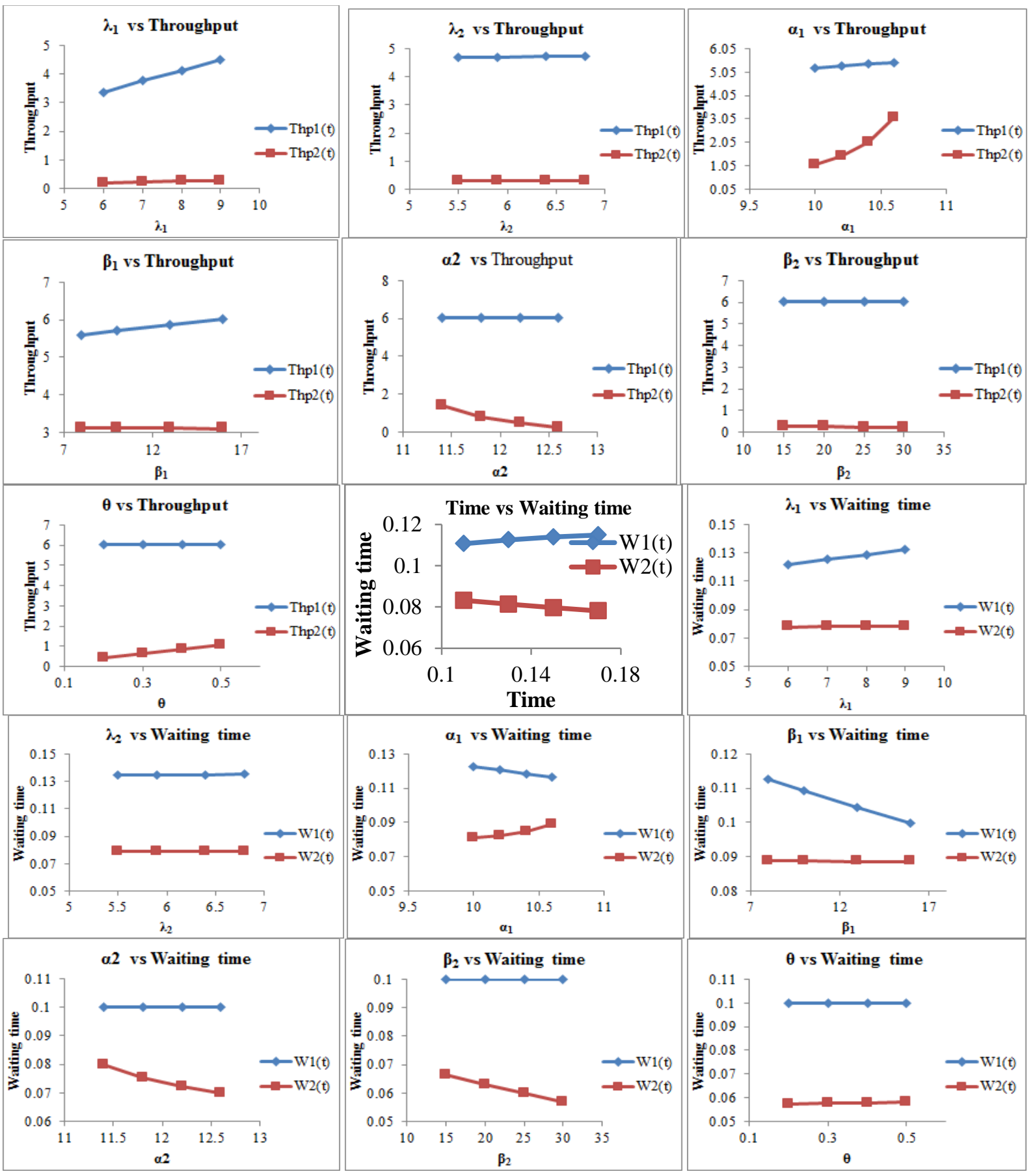

Figure 2b: The relationship between the parameters and performance measures

It is also observed that as the service rate parameter $\left(\beta_{1}\right)$ varies from 8 to 16 , the utilization of the first and second service stations decrease from 0.46728 to 0.45238 and 0.24205 to 0.24172 respectively, the throughput of the first service station increases from 5.58871 to 6.02573 and the second service station decreases from 3.11521 to 3.11092 , and the average waiting time of a customers in the first and second queue decrease from 0.11269 to 0.09993 and 0.08896 to 0.08895 respectively, when all other parameters are fixed. 
International Journal of Research in Advent Technology, Vol.7, No.3, March 2019

\section{E-ISSN: 2321-9637 \\ Available online at www.ijrat.org}

It is observed that as the service rate parameter $\left(\alpha_{2}\right)$ varies from 11.4 to 12.6 , the utilization of the second service station, the throughput of the second service station, and the average waiting time of a customer in the second queue decrease from 0.10614 to $0.01856,1.40847$ to 0.26859 and 0.07966 to 0.06976 respectively.

It is also observed that as the service rate parameter $\left(\beta_{2}\right)$ varies from 15 to 30 , the utilization of the second service station, the throughput of the second service station, and the average waiting time of a customer in the second queue decrease from 0.01709 to $0.01235,0.25897$ to 0.21853 and 0.06658 to 0.05685 respectively. And the performance measures remain constant in first queue, when all other parameters are fixed.

It is also observed that as the service rate parameter $(\theta)$ varies from 0.1 to 0.5 , the utilization of the second service station, the throughput of the second service station, and the average waiting time of a customer in the second queue increase from 0.02454 to $0.06023,0.43437$ to 1.06602 and 0.05720 to 0.05827 respectively. And the performance measures remain constant in first queue, when all other parameters are fixed.

From Table 3 , it is observed that as time (t) varies from 0.11 to 0.17 , the variance of the number of customers in first queue increases from 0.13618 to 0.28872 , in second queue decreases from 0.03125 to 0.00992 and in the system it increases from 0.16743 to 0.29864 , the coefficient of variation of number of customers in first queue decreases from 2.70983 to 1.86106 and in second queue increases from 5.65725 to 10.04078 , when all other parameters are fixed. It is observed that the variance and coefficient of variation of number of customers in each queue are highly sensitive with respect to time.

It is further observed that as the arrival rate parameter $\left(\lambda_{1}\right)$ varies from 6 to 9 , the variance of the number of customers in each queue and in the system increase from 0.41083 to $0.59399,0.01488$ to 0.02232 and 0.42571 to 0.61631 respectively, the coefficient of variation of number of customers in each queue decrease from 1.56016 to 1.29750 and 8.19826 to 6.69385 , when all other parameters are fixed.
It is also observed that as the arrival rate parameter $\left(\lambda_{2}\right)$ varies from 5.5 to 6.8 , the variance of the number of customers in first queue increases from 0.62897 to 0.64054 , in second queue it remains be constant and in the system it increases from 0.65253 to 0.66410 , the coefficient of variation of number of customers in first queue decreases from 1.26091 to 1.24947 and in second queue it remains be constant, when all other parameters are fixed.

It is observed that as the service rate parameter $\left(\alpha_{1}\right)$ varies from 10 to 10.6 , the variance of the number of customers in first queue decreases from 0.64111 to 0.63692 , in second queue increases from 0.08855 to 0.27726 and in the system it increases from 0.72966 to 0.91419 , the coefficient of variation of number of customers in first queue increases from 1.24892 to 1.25302 and in second queue decreases from 3.36057 to 1.89912 , when all other parameters are fixed.

It is also observed that as the service rate parameter $\left(\beta_{1}\right)$ varies from 8 to 16 , the variance of the number of customers in each queue and in the system decrease from 0.62977 to $0.60218,0.27714$ to 0.27670 and 0.90690 to 0.87888 respectively, the coefficient of variation of number of customers in each queue increase from 1.26012 to 1.28866 and 1.89955 to 1.90106 , when all other parameters are fixed.

It is observed that as the service rate parameter $\left(\alpha_{2}\right)$ varies from 11.4 to 12.6 , the variance of the number of customers in the second queue and entire queue decrease from 0.11221 to 0.01874 and 0.71438 to 0.62091 and in first queue they remain constant, the coefficient of variation of number of customers in second queue increases from 2.98533 to 7.30561 and in the first they remain constant, when all other parameters are fixed.

It is also observed that as the service rate parameter $\left(\beta_{2}\right)$ varies from 15 to 30 , the variance of the number of customers in the second queue and entire queue decrease from 0.01724 to $0.01242,0.61942$ to 0.61460 and first queue in they remain constant, the coefficient of variation of number of customers in second queue increases from 7.61575 to 8.97181 and in the first they remain constant, when all other parameters are fixed. 
International Journal of Research in Advent Technology, Vol.7, No.3, March 2019

E-ISSN: 2321-9637

Available online at www.ijrat.org

Table 3

Values of $V_{1}(t), V_{2}(t), V(t), C V_{1}(t), C V_{2}(t)$ for different values of parameters

\begin{tabular}{|c|c|c|c|c|c|c|c|c|c|c|c|c|c|}
\hline $\mathbf{T}$ & $\lambda_{1}$ & $\lambda_{2}$ & $\alpha_{1}$ & $\beta_{1}$ & $\alpha_{2}$ & $\boldsymbol{\beta}_{2}$ & $\Theta$ & $\pi$ & $V_{1}(t)$ & $V_{2}(t)$ & $V(t)$ & $C V_{1}(t)$ & $\mathrm{CV}_{2}(\mathrm{t})$ \\
\hline 0.11 & 4 & 5 & 9 & 6 & 11 & 11 & 0.1 & 0.9 & 0.13618 & 0.03125 & 0.16743 & 2.70983 & 5.65725 \\
\hline 0.13 & 4 & 5 & 9 & 6 & 11 & 11 & 0.1 & 0.9 & 0.19574 & 0.02194 & 0.21768 & 2.26024 & 6.75147 \\
\hline 0.15 & 4 & 5 & 9 & 6 & 11 & 11 & 0.1 & 0.9 & 0.24612 & 0.01501 & 0.26113 & 2.01572 & 8.16255 \\
\hline 0.17 & 4 & 5 & 9 & 6 & 11 & 11 & 0.1 & 0.9 & 0.28872 & 0.00992 & 0.29864 & 1.86106 & 10.04078 \\
\hline 0.17 & 6 & 5 & 9 & 6 & 11 & 11 & 0.1 & 0.9 & 0.41083 & 0.01488 & 0.42571 & 1.56016 & 8.19826 \\
\hline 0.17 & 7 & 5 & 9 & 6 & 11 & 11 & 0.1 & 0.9 & 0.47188 & 0.01736 & 0.48924 & 1.45573 & 7.59011 \\
\hline 0.17 & 8 & 5 & 9 & 6 & 11 & 11 & 0.1 & 0.9 & 0.53294 & 0.01984 & 0.55278 & 1.36981 & 7.09990 \\
\hline 0.17 & 9 & 5 & 9 & 6 & 11 & 11 & 0.1 & 0.9 & 0.59399 & 0.02232 & 0.61631 & 1.29750 & 6.69385 \\
\hline 0.17 & 9.5 & 5.5 & 9 & 6 & 11 & 11 & 0.1 & 0.9 & 0.62897 & 0.02356 & 0.65253 & 1.26091 & 6.51532 \\
\hline 0.17 & 9.5 & 5.9 & 9 & 6 & 11 & 11 & 0.1 & 0.9 & 0.63253 & 0.02356 & 0.65609 & 1.25736 & 6.51532 \\
\hline 0.17 & 9.5 & 6.4 & 9 & 6 & 11 & 11 & 0.1 & 0.9 & 0.63698 & 0.02356 & 0.66054 & 1.25296 & 6.51532 \\
\hline 0.17 & 9.5 & 6.8 & 9 & 6 & 11 & 11 & 0.1 & 0.9 & 0.64054 & 0.02356 & 0.66410 & 1.24947 & 6.51532 \\
\hline 0.17 & 9.5 & 6.8 & 10 & 6 & 11 & 11 & 0.1 & 0.9 & 0.64111 & 0.08855 & 0.72966 & 1.24892 & 3.36057 \\
\hline 0.17 & 9.5 & 6.8 & 10.2 & 6 & 11 & 11 & 0.1 & 0.9 & 0.64002 & 0.12024 & 0.76026 & 1.24998 & 2.88387 \\
\hline 0.17 & 9.5 & 6.8 & 10.4 & 6 & 11 & 11 & 0.1 & 0.9 & 0.63862 & 0.17274 & 0.81135 & 1.25135 & 2.40606 \\
\hline 0.17 & 9.5 & 6.8 & 10.6 & 6 & 11 & 11 & 0.1 & 0.9 & 0.63692 & 0.27726 & 0.91419 & 1.25302 & 1.89912 \\
\hline 0.17 & 9.5 & 6.8 & 10.6 & 8 & 11 & 11 & 0.1 & 0.9 & 0.62977 & 0.27714 & 0.90690 & 1.26012 & 1.89955 \\
\hline 0.17 & 9.5 & 6.8 & 10.6 & 10 & 11 & 11 & 0.1 & 0.9 & 0.62271 & 0.27702 & 0.89973 & 1.26723 & 1.89996 \\
\hline 0.17 & 9.5 & 6.8 & 10.6 & 13 & 11 & 11 & 0.1 & 0.9 & 0.61233 & 0.27685 & 0.88919 & 1.27793 & 1.90053 \\
\hline 0.17 & 9.5 & 6.8 & 10.6 & 16 & 11 & 11 & 0.1 & 0.9 & 0.60218 & 0.27670 & 0.87888 & 1.28866 & 1.90106 \\
\hline 0.17 & 9.5 & 6.8 & 10.6 & 16 & 11.4 & 11 & 0.1 & 0.9 & 0.60218 & 0.11221 & 0.71438 & 1.28866 & 2.98533 \\
\hline 0.17 & 9.5 & 6.8 & 10.6 & 16 & 11.8 & 11 & 0.1 & 0.9 & 0.60218 & 0.05893 & 0.66111 & 1.28866 & 4.11933 \\
\hline 0.17 & 9.5 & 6.8 & 10.6 & 16 & 12.2 & 11 & 0.1 & 0.9 & 0.60218 & 0.03334 & 0.63552 & 1.28866 & 5.47668 \\
\hline 0.17 & 9.5 & 6.8 & 10.6 & 16 & 12.6 & 11 & 0.1 & 0.9 & 0.60218 & 0.01874 & 0.62091 & 1.28866 & 7.30561 \\
\hline 0.17 & 9.5 & 6.8 & 10.6 & 16 & 12.6 & 15 & 0.1 & 0.9 & 0.60218 & 0.01724 & 0.61942 & 1.28866 & 7.61575 \\
\hline 0.17 & 9.5 & 6.8 & 10.6 & 16 & 12.6 & 20 & 0.1 & 0.9 & 0.60218 & 0.01550 & 0.61768 & 1.28866 & 8.03128 \\
\hline 0.17 & 9.5 & 6.8 & 10.6 & 16 & 12.6 & 25 & 0.1 & 0.9 & 0.60218 & 0.0139 & 0.61608 & 1.28866 & 8.48164 \\
\hline 0.17 & 9.5 & 6.8 & 10.6 & 16 & 12.6 & 30 & 0.1 & 0.9 & 0.60218 & 0.01242 & 0.61460 & 1.28866 & 8.97181 \\
\hline 0.17 & 9.5 & 6.8 & 10.6 & 16 & 12.6 & 30 & 0.2 & 0.8 & 0.60218 & 0.02485 & 0.62702 & 1.28866 & 6.34403 \\
\hline 0.17 & 9.5 & 6.8 & 10.6 & 16 & 12.6 & 30 & 0.3 & 0.7 & 0.60218 & 0.03727 & 0.63945 & 1.28866 & 5.17988 \\
\hline 0.17 & 9.5 & 6.8 & 10.6 & 16 & 12.6 & 30 & 0.4 & 0.6 & 0.60218 & 0.04969 & 0.65187 & 1.28866 & 4.48590 \\
\hline 0.17 & 9.5 & 6.8 & 10.6 & 16 & 12.6 & 30 & 0.5 & 0.5 & 0.60218 & 0.06212 & 0.66429 & 1.28866 & 4.01231 \\
\hline
\end{tabular}

It is also observed that as the service rate parameter $(\theta)$ varies from 0.2 to 0.5 , the variance of the number of customers in the second queue and entire queue increase from 0.02485 to $0.06212,0.62702$ to 0.66429 and first queue in they remain constant, the coefficient of variation of number of customers in second queue decreases from 6.34403 to 4.01231 and in the first they remain constant, when all other parameters are fixed.

\section{SENSITIVITY ANALYSIS OF THE MODEL}

Sensitivity analysis of the model is performed with respect to the value of time $(t)$, arrival rate $\lambda(t)$, service rates of the first, second servers $\mu_{1}(t), \mu_{2}(t)$ and all parameters together on the performance measures such as mean number of customers in the first, second queues, the utilization of service station in first, second queues, the mean delay in the first, second queues and the through put of service station in first and second queues.

For different values of $t, \lambda_{1}, \lambda_{2}, \alpha_{1}, \beta_{1}, \alpha_{2}, \beta_{2}$ and $\theta$ the mean number of customers in the first and second queue, the utilization of service station in first and second queue, the mean delay in the first and second queue and the throughput of service station in first and second queue are computed with variation of $-10 \%,-5 \%, 0 \%, 5 \%, 10 \%$, are given in table 4 . The following data has been considered for the sensitivity 
International Journal of Research in Advent Technology, Vol.7, No.3, March 2019

E-ISSN: 2321-9637

Available online at www.ijrat.org

analysis: $\mathrm{t}=0.2 \quad \lambda_{1}=4 \quad \lambda_{2}=6 \quad \alpha_{1}=9 \quad \beta_{1}=5$ $\alpha_{2}=10.5 \quad \beta_{2}=10$ and $\theta=0.1$

The sensitivity analysis for the mean number of customers, the utilization of service stations, the mean delay in transmission, and the throughput are computed and presented in Table 4 with variation of $10 \%,-5 \%, 0 \%, 5 \%, 10 \%$, on the model parameters. The performance measure are highly affected by time (t). As t increases from $-10 \%$ to $+10 \%$, the average number of customers in the first queue increases along with the average delay, the utilization and the throughput of the first service stations. The average number of customers in the second queue decreases along with the average delay, the utilization and the throughput of the second service stations.

Arrival rate parameter $\lambda_{1}$ increases $-10 \%$ to $+10 \%$, the average number of customers in each queue increase along with the average delay in transmission, the utilization of service stations and throughput of each service stations.

Arrival rate parameter $\lambda_{2}$ increases $-10 \%$ to $+10 \%$, the average number of customers in the first queue increase along with the average delay, the utilization of first node and the throughput of the first service stations and there is no change with respect to the performance measures of second queue.

Service rate parameter $\alpha_{1}$ increases from $-10 \%$ to $+10 \%$, the average number of customers in the first queue decrease along with the average delay, the average number of customers in the second queue increase along with the average delay, the utilization of first service stations decreases and in second service stations it increases, the throughput of the each service stations is increasing.

Service rate parameter $\beta_{1}$ increases from $-10 \%$ to $+10 \%$, the average number of customers in each queue is decreasing along with the average delay in each queue, the utilization of each service stations decreases. The throughput of the first service stations increases and in the second service stations it decreases.

Service rate parameters $\alpha_{2}$ and $\beta_{2}$ increase from $-10 \%$ to $+10 \%$, the average number of customers in the second queue decreases along with the average delay in the second queue, the utilization and throughput of the second service station decrease and there is no change with respect to the performance measures of first queue. Service rate parameter $\theta$ increases from $-10 \%$ to $+10 \%$, the average number of customers in the second queue increase along with the average delay in the second queue, the utilization and throughput of the second service station increase and there is no change with respect to the performance measures of first queue

Table 4

The Values of $L_{1}(t), L_{2}(t), U_{1}(t), U_{2}(t), T_{h} p_{1}(t), T_{h} p_{2}(t), W_{1}(t), W_{2}(t)$ for different values of $t, \lambda_{11}, \lambda_{12}, \lambda_{21}, \lambda_{22}$, $\alpha_{1}, \beta_{1}, \alpha_{2}, \beta_{2}, \theta$ parameters

\begin{tabular}{|c|c|c|c|c|c|c|c|c|c|}
\hline \multirow[t]{2}{*}{ Parameter } & \multirow{2}{*}{$\begin{array}{l}\% \text { change in } \\
\text { parameters }\end{array}$} & \multicolumn{8}{|c|}{ Performance Measures } \\
\hline & & $\mathrm{L}_{1}(\mathrm{t})$ & $\mathbf{L}_{2}(t)$ & $\mathrm{U}_{1}(\mathbf{t})$ & $\mathbf{U}_{2}(\mathbf{t})$ & $\mathbf{W}_{1}(\mathbf{t})$ & $\mathbf{W}_{2}(\mathbf{t})$ & $\operatorname{Thp}_{1}(\mathrm{t})$ & $\operatorname{Thp}_{2}(\mathrm{t})$ \\
\hline \multirow[t]{5}{*}{$t=0.2$} & $-10 \%$ & 0.31916 & 0.01785 & 0.27324 & 0.01770 & 0.11799 & 0.08203 & 2.70506 & 0.21766 \\
\hline & $-5 \%$ & 0.33765 & 0.01506 & 0.28656 & 0.01495 & 0.11842 & 0.08125 & 2.85123 & 0.18534 \\
\hline & $0 \%$ & 0.35480 & 0.01264 & 0.29869 & 0.01256 & 0.11879 & 0.08051 & 2.98689 & 0.15703 \\
\hline & $+5 \%$ & 0.37073 & 0.01056 & 0.30977 & 0.01050 & 0.11908 & 0.07978 & 3.11316 & 0.13230 \\
\hline & $+10 \%$ & 0.38552 & 0.00876 & 0.31990 & 0.00872 & 0.11932 & 0.07909 & 3.23102 & 0.11077 \\
\hline \multirow[t]{5}{*}{$\lambda_{1}=4$} & $-10 \%$ & 0.32620 & 0.01138 & 0.27834 & 0.01131 & 0.11720 & 0.08046 & 2.78340 & 0.14141 \\
\hline & $-5 \%$ & 0.34050 & 0.01201 & 0.28859 & 0.01194 & 0.11799 & 0.08048 & 2.88587 & 0.14922 \\
\hline & 0\% & 0.35480 & 0.01264 & 0.29869 & 0.01256 & 0.11879 & 0.08051 & 2.98689 & 0.15703 \\
\hline & $+5 \%$ & 0.36910 & 0.01327 & 0.30865 & 0.01319 & 0.11959 & 0.08053 & 3.08647 & 0.16483 \\
\hline & $+10 \%$ & 0.38341 & 0.01391 & 0.31846 & 0.01381 & 0.12039 & 0.08056 & 3.18463 & 0.17262 \\
\hline \multirow[t]{5}{*}{$\lambda_{2}=6$} & $-10 \%$ & 0.34792 & 0.01264 & 0.29385 & 0.01256 & 0.11840 & 0.08051 & 2.93848 & 0.15703 \\
\hline & $-5 \%$ & 0.35136 & 0.01264 & 0.29627 & 0.01256 & 0.11859 & 0.08051 & 2.96272 & 0.15703 \\
\hline & 0\% & 0.35480 & 0.01264 & 0.29869 & 0.01256 & 0.11879 & 0.08051 & 2.98689 & 0.15703 \\
\hline & $+5 \%$ & 0.35824 & 0.01264 & 0.30110 & 0.01256 & 0.11898 & 0.08051 & 3.01096 & 0.15703 \\
\hline & $+10 \%$ & 0.36168 & 0.01264 & 0.30350 & 0.01256 & 0.11917 & 0.08051 & 3.03496 & 0.15703 \\
\hline \multirow[t]{2}{*}{$\alpha_{1}=9$} & $-10 \%$ & 0.35927 & 0.00147 & 0.30182 & 0.00147 & 0.13081 & 0.08006 & 2.74653 & 0.01842 \\
\hline & $-5 \%$ & 0.35761 & 0.00592 & 0.30065 & 0.00590 & 0.12455 & 0.08024 & 2.87122 & 0.07381 \\
\hline
\end{tabular}


International Journal of Research in Advent Technology, Vol.7, No.3, March 2019

E-ISSN: 2321-9637

Available online at www.ijrat.org

\begin{tabular}{|c|c|c|c|c|c|c|c|c|c|}
\hline & 0\% & 0.35480 & 0.01264 & 0.29869 & 0.01256 & 0.11879 & 0.08051 & 2.98689 & 0.15703 \\
\hline & $+5 \%$ & 0.35113 & 0.02461 & 0.29611 & 0.02431 & 0.11348 & 0.08099 & 3.09434 & 0.30389 \\
\hline & $+10 \%$ & 0.34680 & 0.05373 & 0.29305 & 0.05232 & 0.10857 & 0.08217 & 3.19429 & 0.65395 \\
\hline \multirow[t]{5}{*}{$\beta_{1}=5$} & $-10 \%$ & 0.35619 & 0.01266 & 0.29966 & 0.01258 & 0.12007 & 0.08051 & 2.96665 & 0.15726 \\
\hline & $-5 \%$ & 0.35550 & 0.01265 & 0.29917 & 0.01257 & 0.11942 & 0.08051 & 2.97679 & 0.15714 \\
\hline & 0\% & 0.35480 & 0.01264 & 0.29869 & 0.01256 & 0.11879 & 0.08051 & 2.98689 & 0.15703 \\
\hline & $+5 \%$ & 0.35411 & 0.01263 & 0.29820 & 0.01255 & 0.11816 & 0.08051 & 2.99695 & 0.15691 \\
\hline & $+10 \%$ & 0.35342 & 0.01262 & 0.29772 & 0.01254 & 0.11753 & 0.08051 & 3.00697 & 0.15680 \\
\hline \multirow[t]{5}{*}{$\alpha_{2}=10.5$} & $-10 \%$ & 0.35480 & 0.09338 & 0.29869 & 0.08915 & 0.11879 & 0.09148 & 2.98689 & 1.02076 \\
\hline & $-5 \%$ & 0.35480 & 0.03043 & 0.29869 & 0.02998 & 0.11879 & 0.08478 & 2.98689 & 0.35896 \\
\hline & $0 \%$ & 0.35480 & 0.01264 & 0.29869 & 0.01256 & 0.11879 & 0.08051 & 2.98689 & 0.15703 \\
\hline & $+5 \%$ & 0.35480 & 0.00476 & 0.29869 & 0.00475 & 0.11879 & 0.07696 & 2.98689 & 0.06183 \\
\hline & $+10 \%$ & 0.35480 & 0.00058 & 0.29869 & 0.00058 & 0.11879 & 0.07382 & 2.98689 & 0.00791 \\
\hline \multirow[t]{5}{*}{$\beta_{2}=10$} & $-10 \%$ & 0.35480 & 0.01298 & 0.29869 & 0.01289 & 0.11879 & 0.08183 & 2.98689 & 0.15857 \\
\hline & $-5 \%$ & 0.35480 & 0.01281 & 0.29869 & 0.01273 & 0.11879 & 0.08116 & 2.98689 & 0.15780 \\
\hline & 0\% & 0.35480 & 0.01264 & 0.29869 & 0.01256 & 0.11879 & 0.08051 & 2.98689 & 0.15703 \\
\hline & $+5 \%$ & 0.35480 & 0.01248 & 0.29869 & 0.01240 & 0.11879 & 0.07986 & 2.98689 & 0.15624 \\
\hline & $+10 \%$ & 0.35480 & 0.01232 & 0.29869 & 0.01224 & 0.11879 & 0.07923 & 2.98689 & 0.15544 \\
\hline \multirow[t]{5}{*}{$\theta=0.1$} & $-10 \%$ & 0.35480 & 0.01138 & 0.29869 & 0.01131 & 0.11879 & 0.08046 & 2.98689 & 0.14141 \\
\hline & $-5 \%$ & 0.35480 & 0.01201 & 0.29869 & 0.01194 & 0.11879 & 0.08048 & 2.98689 & 0.14922 \\
\hline & $0 \%$ & 0.35480 & 0.01264 & 0.29869 & 0.01256 & 0.11879 & 0.08051 & 2.98689 & 0.15703 \\
\hline & $+5 \%$ & 0.35480 & 0.01327 & 0.29869 & 0.01319 & 0.11879 & 0.08053 & 2.98689 & 0.16483 \\
\hline & $+10 \%$ & 0.35480 & 0.01391 & 0.29869 & 0.01381 & 0.11879 & 0.08056 & 2.98689 & 0.17262 \\
\hline
\end{tabular}

\section{COMPARITIVE STUDY}

The comparative study of the developed model with that of homogeneous poison arrival and service rates is performed by computing the performance measure. The results are presented in the Table 5 for different values of $\mathrm{t}=0.18,0.20,0.22,0.24$.

From Table 5, it is observed that as the time increases the percentage variation of the performance measures between the two models also increasing. It is observed that the assumption of non-homogeneous Poisson arrival and service processes has a significant influence on all the performance measures of the queuing model. Also time has a significant effect on the system performance and the proposed model can predict the performance measures more accurately.

Table 5

Comparative study of models with Non-Homogeneous and Homogeneous Poisson service rates

\begin{tabular}{|c|c|c|c|c|c|}
\hline $\mathbf{T}$ & $\begin{array}{l}\text { Parameter } \\
\text { Measure }\end{array}$ & $\begin{array}{l}\text { Models with } \\
\text { Non-Homogeneous } \\
\text { arrival rate }\end{array}$ & $\begin{array}{l}\text { Models with Homogeneous } \\
\text { arrival rate }\end{array}$ & Difference & $\begin{array}{l}\text { Percentage of } \\
\text { Variation }\end{array}$ \\
\hline \multirow[t]{6}{*}{0.18} & $\mathbf{L}_{1}(\mathbf{t})$ & 0.62670 & 0.63027 & 0.00357 & 0.566424 \\
\hline & $\mathbf{L}_{2}(\mathbf{t})$ & 0.01344 & 0.02006 & 0.00662 & 33.001 \\
\hline & $\mathrm{U}_{1}(\mathbf{t})$ & 0.46565 & 0.46755 & 0.0019 & 0.406374 \\
\hline & $\mathrm{U}_{2}(\mathbf{t})$ & 0.01335 & 0.01986 & 0.00651 & 32.77946 \\
\hline & $W_{1}(t)$ & 0.09984 & 0.12717 & 0.02733 & 21.49092 \\
\hline & $W_{2}(t)$ & 0.06580 & 0.08016 & 0.01436 & 17.91417 \\
\hline \multirow[t]{6}{*}{0.20} & $\mathbf{L}_{1}(\mathbf{t})$ & 0.66593 & 0.68108 & 0.01515 & 2.224408 \\
\hline & $\mathbf{L}_{2}(\mathbf{t})$ & 0.00778 & 0.01336 & 0.00558 & 41.76647 \\
\hline & $\mathrm{U}_{1}(\mathbf{t})$ & 0.48620 & 0.49393 & 0.00773 & 1.564999 \\
\hline & $U_{2}(t)$ & 0.00775 & 0.01327 & 0.00552 & 41.59759 \\
\hline & $W_{1}(t)$ & 0.09925 & 0.13008 & 0.03083 & 23.7008 \\
\hline & $\mathbf{W}_{2}(\mathbf{t})$ & 0.06435 & 0.07990 & 0.01555 & 19.46183 \\
\hline 0.22 & $\mathbf{L}_{1}(\mathbf{t})$ & 0.69436 & 0.72218 & 0.02782 & 3.852225 \\
\hline
\end{tabular}


International Journal of Research in Advent Technology, Vol.7, No.3, March 2019

E-ISSN: 2321-9637

Available online at www.ijrat.org

\begin{tabular}{|c|l|c|c|c|c|}
\hline \multirow{7}{*}{} & $\mathbf{L}_{\mathbf{2}}(\mathbf{t})$ & 0.00406 & 0.00857 & 0.00451 & 52.62544 \\
\cline { 2 - 6 } & $\mathbf{U}_{\mathbf{1}}(\mathbf{t})$ & 0.50060 & 0.51431 & 0.01371 & 2.665707 \\
\cline { 2 - 6 } & $\mathbf{U}_{\mathbf{2}}(\mathbf{t})$ & 0.00405 & 0.00854 & 0.00449 & 52.57611 \\
\cline { 2 - 6 } & $\mathbf{W}_{\mathbf{1}}(\mathbf{t})$ & 0.09823 & 0.13247 & 0.03424 & 25.84736 \\
\cline { 2 - 6 } & $\mathbf{W}_{\mathbf{2}}(\mathbf{t})$ & 0.06302 & 0.07971 & 0.01669 & 20.9384 \\
\hline \multirow{0}{0.24}{} & $\mathbf{L}_{\mathbf{1}}(\mathbf{t})$ & 0.71429 & 0.75543 & 0.04114 & 5.445905 \\
\cline { 2 - 6 } & $\mathbf{L}_{\mathbf{2}}(\mathbf{t})$ & 0.00170 & 0.00520 & 0.0035 & 67.30769 \\
\cline { 2 - 6 } & $\mathbf{U}_{\mathbf{1}}(\mathbf{t})$ & 0.51046 & 0.53019 & 0.01973 & 3.721307 \\
\cline { 2 - 6 } & $\mathbf{U}_{\mathbf{2}}(\mathbf{t})$ & 0.00169 & 0.00519 & 0.0035 & 67.43738 \\
\cline { 2 - 6 } & $\mathbf{W}_{\mathbf{1}}(\mathbf{t})$ & 0.09690 & 0.13442 & 0.03752 & 27.91251 \\
\cline { 2 - 6 } & $\mathbf{W}_{\mathbf{2}}(\mathbf{t})$ & 0.06178 & 0.07957 & 0.01779 & 22.35767 \\
\hline
\end{tabular}

\section{CONCLUSION:}

This paper addresses a novel queuing model in which two queues are connected in tandem and having phase type service with time dependent arrival and service processes. It is further assumed the service rates in each service station is dependent on content of the buffers connected to it. The intermediate departures after first service station can reduce the burstness in buffers and delay in transmission. It is also observed that the nonstationary assumptions made for arrival and service processes can capture the non-smooth and bursty traffic of network. The developed model can be utilized for performance evaluation of communication networks such as LAN, MAN and VAN in which the traffic is non-smoother and bursty. This model also includes some of the earlier queuing models as particular cases for specific values of the parameters. It is possible to develop tandem queuing models with bulk arrivals having time dependent arrival and service processes. It is also possible to carry out estimation procedures for estimating the parameters involved in the model these investigation will be taken in future.

\section{REFERENCES:}

[1.] A.V.S Suhasini, K. Srinivasa Rao and P.R.S Reddy.(2013), "On parallel and series Nonhomogeneous bulk arrival queueing model". OPSEARCH 50(4),521-547.

[2.] Choi, B.D.and Choi, D.I (1996),"Queuing system with length dependence service times and its applications to cell discarding scheme in ATM networks", IEEE proceedings communications, Vol.143, pp:5-11.

[3.] Duraga Aparajitha J, G.V.S. Rajkumar(2015), "Single Server Queuing model with time and state dependent service rate", Journal of the Indian Society for Probability and Statistics Vol.15,67-78.

[4.] Davis, J.L., Massey W.A., and Whitty, W., (1995) "Sensitivity to the service- time distribution in the non- stationary Erlang loss model ${ }^{e e}$. Management Science. Vol.41, No 6, PP 1107-1116.

[5.] Duffield, N.G., Massey, W.A., and Whitt,W., (2001) " A nonstationary offered load model for packet networks"e. Telecommunication systems. Vol.13, Issue 3/4, PP 271- 296.

[6.] Durga aparajitha J.,Rajkumak G.V.S, (2014) "single server queueing model with time and state dependent service rate". Journal of the Indian society for probability and statistics. Vol.15, PP 67-77.

[7.] Erlang, A.K.(1909),'Probability and telephone calls", Nyt. Tidsskr Krarup Mat.Ser.B, Vol.20,33-39.

[8.] Kin K.Leung (2002), “ Load-dependent service queues with application to congestion control in broadband networks", Performance Evaluation, Vol.50, No4,pp:27-40.

[9.] M.V. Ramasundari, K. Srinivasa Rao, P. Srinivasa Rao and P. Suresh Varma (2011), "Three node communication network model with dynamic bandwidth allocation and Non-homogeneous Poisson arrivals", International journal of Computer Applictions, Vol. 7, No.1,pp:19-27.

[10.] Mandelbaum, A. and Massey, W.A., (1995) " Strong approximations for time dependent queues". MOR. Vol.20, No1, PP 33-64.

[11.] Massey, W.A. and Whitt, W. (1993), "Networks of infinite-server queues with nonstationary Poisson input queueing systems". Queueing systems and their applications. Vol.13, No1, PP 183-250. 
International Journal of Research in Advent Technology, Vol.7, No.3, March 2019

\author{
E-ISSN: 2321-9637 \\ Available online at www.ijrat.org
}

[12.] Massey, W.A. and Whitt, W. (1994), “An analysis of the modified load approximation for the nonstationary Erlang loss model". Annals of applied probability Vol.4, No 4, PP 1145-1160.

[13.] Newell, G.F. (1968), "Queues with timedependent arrival rates (parts I-III) ${ }^{\circ e}$. Journal of Applied probability. Vol.5, PP 436-451(I), 579-590 (II), 591- 606 II).

[14.] Nageswararao, K., Srinivasa Rao, K. an Srinivasarao, P. (2010), "A tandom communication net work with dynamic band width allocation and modified phase type transmission having bulk arrivals", International journal of Computer Sciences, Issues, Vol.7,No.2,pp:18-26.

[15.] Padmavathi, G., Srinivasa Rao, K and Reddy, K.V.V.S (2009), "Performance Evaluation of parallel and series communication network with dynamic band width allocation CIIT" International journal of Networking and Communication, Vol.1, No.7,pp:433-454.

[16.] P. Trinath Rao, K. Srinivasa Rao and K.V.V.S Reddy(2012), "Performance of Non-homogeneous communication with Poisson arrivals and dynamic bandwidth allocation" accepted in International Journal of Systems, control and communication, Vol.4(3),164-182.

[17.] Parthasarathy, P.R and Selvaraju, N. (2001)," Transient analysis of queue where potential customers are discouraged by queue length", Mathematical problem in Engineering, Vol. 7,pp: 433-454.

[18.] Srinivasa Rao, K. Shoba,T. Srinivasa Rao, P.(2000), "The $M / M / 1$ interdependent queuing model with controllable arrival rates", Opsearch Vol 37(1) pp:14-24.

[19.] Srinivasa Rao, K. Srinivasa Rao, P. Lakshminarayana , j. ” $\mathrm{M} / \mathrm{M} / 1$ interdependent queueing model with servers vacaion" Proc.of AP Akademi of Sciences Vol7, No.3(2003),pp:191-196.

[20.] Srinivasa Rao, K., Suresh varma, P. and Srinivas, Y.(2008), "Interdependent Queuing model with startup delay". Statistical theory and App.7(2), 219-228.

[21.] Srinivasa Rao, K. and Vijay kumar CVRS (2003), " interdependent tandem queuing model". International journal of Management and Systems Vol 16(2) pp:157-168.
[22.] Suresh Varma, P. Srinivasa Rao, K.(2007), “ A communication Network with load dependent transmission", International Journal of Mathematical Sciences, Issues, Vol.7, No.2,pp:199-210.

[23.] Suhasini, A.V.S, K. Srinivasa Rao, P.R.S. Reddy.(2014),"Queuing model with Nonhomogeneous bulk arrivals having state dependent service rates" int. J. Operation research Vol:21(1),8499.

[24.] Suhasini, A.V.S, K. Srinivasa Rao, P.R.S. Reddy.(2013)," Transient analysis of parallel and series queuing model with Non-homogeneous Poisson binomial bulk arrival and load dependent service rates" Neural , parallel and scientific computations, Vol:21(2),235-262.

[25.] Suhasini, A.V.S, K. Srinivasa Rao, P.R.S. Reddy.(2012)," Transient analysis of tandem queuing model with Non-homogeneous Poisson bulk arrivals having state dependent service rates", Int.J.Adv.Comp.math.Sci.Vol:3(3),272-289.

[26.] Sadu, A. R., Srinivasa rao, K., Nirupama devi, $\mathrm{K}$, "Forked queueing model with load dependent service rate bulk arrivals". International journal of operation research, Vol.30, No1, PP 1-32.

[27.] Srinivasa Rao, K., M.Govinda Rao and K.Naveen Kuamr (2011) - Transient analysis of an interdependent forked Tandem queueing model with load dependent service rate, International Journal of Computer Applications (IJCA), Volume 34, No. 3, pp: $33-40$.

[28.] Srinivasa Rao,K., VAsanta, M.R., and Vijaya Kumar, C. V. R.S., (2000), on an interdependent communication Network, opsearch.37(2):134-143.

[29.] Srinivasa Rao. K, J. Durga aparajitha (2017) “ Two node tandem queuing model with phase type state and time dependent service rates" International Journal of Computer Applications (0975 - 8887) Volume 177 - No.3, November 2017

[30.] Ward Whitt (2016), "Recent papers on the timevarying single-server queue".//http.pdfs.semanticsscholar.org//

[31.] William A. Massey (1996), "Stability for queues with time varying rates". Stochastic Networks of the series lecture notes in statistics 117: 95-107.

[32.] William A. Massey (2002), "The analysis of queues with time varying rates for telecommunication models". Telecommunication system 21:2-4,173-204. 